\title{
DOLBEAULT HOMOTOPY THEORY
}

\author{
BY \\ JOSEPH NEISENDORFER AND LAURENCE TAYLOR ${ }^{1}$
}

\begin{abstract}
For complex manifolds, we define "complex homotopy groups" in terms of the Dolbeault complex. Many theorems of classical homotopy theory are reflected in the properties of complex homotopy groups. Analytic fibre bundles yield long exact sequences of complex homotopy groups and various Hurewicz theorems relate complex homotopy groups to the Dolbeault cohomology. In a more analytic vein, the classical Fröhlicher spectral sequence has a complex homotopy analogue.

We compute these complex homotopy invariants for such examples as Calabi-Eckmann manifolds, Stein manifolds, and complete intersections.
\end{abstract}

1. Introduction. Sullivan [15] has shown that the de Rham complex of a manifold determines all of the real homotopy invariants. The underlying theme of this paper is to define, for complex manifolds, "complex homotopy invariants" using the Dolbeault complex. This is not an empty exercise. The resulting theory can be used in two ways. Just as ordinary homotopy theory aids in the computation of cohomology, Dolbeault homotopy can be used to help calculate Dolbeault cohomology. As an example, we completely analyze the Calabi-Eckmann manifolds without the use of spectral sequences. In the other direction, there is a relationship between Dolbeault homotopy and ordinary homotopy. In nice cases, such as Kähler manifolds, given information about the Dolbeault homotopy, we can prove results about real, rational, and even integral homotopy. For example, the rank of the integral Hurewicz homomorphism in odd dimensions is even for a compact Kähler manifold. Even though this latter sort of theorem can be proved using the theory in [6] (after passing to the complex numbers), we feel that they occur more naturally in our formalism.

We begin the real work of this paper in $\$ 2$ by defining Dolbeault cohomotopy groups from the Dolbeault complex in a manner reminiscent of Sullivan's definition of de Rham cohomotopy groups. The Dolbeault cohomotopy groups, like the Dolbeault cohomology groups, are bigraded.

Received by the editors June 29, 1977.

AMS (MOS) subject classifications (1970). Primary 32C10, 55E05; Secondary 55H20, 32C35, 32L05, 55E15.

Key words and phrases. Rational homotopy theory, Dolbeault cohomology, complex manifolds, Fröhlicher spectral sequence, Hurewicz theorem, Kähler manifold, Whitehead products.

'Second author partially supported by NSF grants MPS 72-04756 A03 and MCS 76-07158 A01.

() American Mathematical Society 1979 
They are a bit peculiar in that, while the total degree is positive, the second coordinate of the bidegree may be arbitrarily small negative.

Sullivan's theory is really a homotopy theory for differential graded algebras such as the de Rham complex. The Dolbeault complex is bigraded, so we develop an analogous theory for differential bigraded algebras. Actually, we make a few changes of notation and proceed to let the paper of Bousfield and Gugenheim [3] bear the brunt of the work.

The Dolbeault complex and the de Rham complex are naturally related. This fact will enable us to pass back through Sullivan's theory and obtain honest homotopy information about complex manifolds.

Fröhlicher [8] constructed a spectral sequence from the Dolbeault cohomology to the de Rham. We get a similar spectral sequence for cohomotopy if the complex manifold has no nonconstant holomorphic functions.

When the complex manifold has nonconstant holomorphic functions, there can be no Fröhlicher spectral sequence for cohomotopy. We illustrate this by computing the Dolbeault cohomotopy groups of an arbitrary Stein manifold.

Just as ordinary homotopy behaves well with respect to fibrations, Dolbeault cohomotopy behaves well with respect to analytic fibre spaces with some annoying technical restrictions. Spectral sequences also play an important part in the ordinary theory of fibrations. As a consequence of Borel's Serre spectral sequence for Dolbeault cohomology, we derive an EilenbergMoore type spectral sequence.

An important subset of Dolbeault complex is the set of forms of type $(p, 0)$ for which $\bar{\partial}=0$, the holomorphic $p$-forms. An even more important subset for us is the set of all forms of type $(0, q)$. We will, by abuse of the English language, refer to these as antiholomorphic $q$-forms. The importance of the antiholomorphic forms is related to the fact that they form a differential, bigraded, subalgebra retract of the Dolbeault complex.

The bigraded structure on our complexes allows for Hurewicz theorems which are stronger than the ones for de Rham theory. In particular, we prove a strong antiholomorphic Hurewicz theory (Theorem 5) and somewhat weaker holomorphic and sweep Hurewicz theorems (Theorems 6 and 7).

When the complex manifold has no nonconstant holomorphic functions, Dolbeault cohomotopy has the additional structure of a Whitehead coalgebra, just as the de Rham cohomotopy does. The dual of a Whitehead coalgebra is, of course, a Whitehead algebra (under a finiteness condition). This beasty looks much like a graded Lie algebra. Formally they are related by regrading and changing the sign by $(-1)$ to the degree of the first factor [22, formula 4.5].

One use of this theory is to the study of Kähler manifolds. Deligne, Griffiths, Morgan, and Sullivan [6] have shown that the $d d^{c}$-lemma (or its 
equivalent, the $\partial \bar{\partial}$-lemma) forces the real homotopy type of a Kähler manifold from knowledge of its de Rham cohomology ring. Of course, using their results and techniques we can prove that the "complex homotopy type" of a Kähler manifold is a formal consequence of its Dolbeault cohomology ring. One example of this is Theorem 10 where we show that the complex homotopy type of a pull-back of analytic fibre bundles

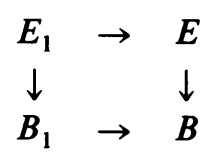

is just $B\left(H^{*},{ }^{*}\left(B_{1}\right), H^{*},{ }^{*}(B), H^{*},{ }^{*}(E)\right.$ ) (the two-sided bar construction on the Dolbeault cohomology rings) where $E, B$, and $B_{1}$ are Kähler.

Another example of the use of formality is Theorem 11, where we prove that the antiholomorphic cohomotopy is a formal consequence of the antiholomorphic cohomology, and that the holomorphic cohomology generates what we call a formal fan in cohomotopy.

For compact Kähler manifolds, there is a "Hodge decomposition" of real homotopy type tensored with $C$. We can use this and Theorem 11 to show the existence of huge free integral Whitehead algebras in the ordinary homotopy of complete intersections.

A more subtle use of the Hodge decomposition enables us to give an elementary computation of the rational homotopy groups of complete intersections (Theorem 14).

2. Basic definitions and formality. Throughout, $M^{n}$ is a complex manifold of complex dimension $n$.

$\mathcal{E}_{M}^{p, q}$ is the space of complex valued differential forms of type $(p, q)$ on $M$. $\mathcal{E}_{M}^{* * *}=\bigoplus \mathcal{E}_{m}^{p, q}$ for all $p \geqslant 0, q \geqslant 0 . \mathcal{E}_{M}^{r}=\bigoplus_{p+q=r} \mathcal{E}_{M}^{p, q}$ and $\mathcal{E}_{M}^{*}=\bigoplus \mathcal{E}_{M}^{r}$ for all $r$.

We define a differential bigraded algebra (DBA) to be a bigraded commutative algebra over $\mathbf{C}$ with a differential of type $(0,1)$ which is a derivation, i.e. $\bar{\partial}(a b)=(\bar{\partial} a) b+(-1)^{\operatorname{deg} a} a(\bar{\partial} b)$ where $\operatorname{deg} a$ is the total degree of $a$. We further require that it be augmented over $\mathbf{C}$. We have the category of DBA's by requiring the maps to be bidegree preserving algebra maps which commute with the differential. There is also a category of DGA's, augmented, differential graded commutative algebras. We finally stipulate that DBA's have nonnegative total degree and that DGA's have nonnegative degree.

Now $\mathcal{E}_{M}^{* * *}$ with the $\bar{\partial}$ differential is a DBA. Its cohomology, denoted $H^{*, *}(M)$, is the classical Dolbeault cohomology [10], [21]. $\mathcal{E}_{M}^{* * *}$ is augmented by choosing a point $p$ in $M$ and evaluating the $C^{\infty}$ complex valued functions at $p$. Since these functions are $\mathcal{E}_{M}^{0,0}$, this augments $\mathcal{E}_{M}^{*, *}$. This makes complex homotopy theory depend on the choice of basepoint, as it should. Clearly, $\mathcal{E}_{M}^{*}$ 
with the $d$ differential is a DGA. Its cohomology is denoted $H^{*}(M)$ and is the classical cohomology of $\boldsymbol{M}$ with complex coefficients.

$\mathcal{E}_{M}^{* *}$ has another differential, $\partial$, of type $(1,0)$. We define a BBA (bidifferential, bigraded algebra) to be a DBA with another differential $\partial$ of type $(1,0)$. We further require that $\partial$ is a derivation and that $\overline{\partial \partial}=-\partial \bar{\partial}$. When we speak of the cohomology of a BBA we are always speaking of cohomology (homology actually) with respect to the $\bar{\partial}$ differential, which we of ten speak of as the distinguished differential. $\mathcal{E}_{M}^{* * *}$ is a BBA.

Given two BBA's, DBA's, or DGA's and a map between them, we say that the map is a weak equivalence if it induces an isomorphism in cohomology. A BBA map must commute with the $\partial$ differential, but, to be a weak equivalence, need only induce an isomorphism in $\bar{\partial}$ cohomology.

In Sullivan's theory, the basic building blocks are the minimal models [6]. Bousfield and Gugenheim [3] produce a more functorial theory using cofibrant models. We define a cofibrant DBA as follows. $\mathbb{Q}$ is cofibrant if given any map of DBA's $g: \mathscr{B} \rightarrow \mathcal{C}$ which is both a surjection and a weak equivalence, and given any map $f: \mathbb{Q} \rightarrow \mathcal{C}$ (of DBA's of course), then there exists $h: \mathbb{Q} \rightarrow \mathscr{B}$ such that $g \circ h=f$. A cofibrant model for a DBA, $\mathscr{D}$, is a cofibrant DBA, $\mathscr{N}$, and a weak equivalence $g: \Re \rightarrow \mathscr{D}$.

By $Q(\mathbb{Q})$ we mean the indecomposables of the DGA (DBA or BBA) $\mathbb{Q}$. Alternatively, if $\bar{Q}$ denotes the kernel of the augmentation, $Q(\mathbb{Q})$ is the cokernel of the product map $\overline{\mathbb{Q}} \otimes \bar{Q} \rightarrow \bar{Q} . Q(\mathbb{Q})$ is a graded (bigraded) vector space with a differential.

Now let $\mathbb{Q}$ be a DBA. $\pi^{*, *}(\mathscr{Q})$ is defined as follows. Pick a cofibrant model, $\mathfrak{T}$, for $\mathcal{Q}$, and let $\pi^{*, *}(\mathscr{Q})=H^{*, *}(Q(\mathfrak{T}))$. In $\$ 3$ we will show that $\pi^{*, *}(\mathscr{Q})$ is defined ( $\pi$ exists) and even that $\pi^{* * *}(\mathscr{Q})$ is well defined and functional. If $\mathcal{Q}$ is only a DGA, $\pi^{*}(\mathcal{Q})$ is defined similarly, and the cohomotopy of a BBA is obtained by forgetting the extra differential.

The Dolbeault cohomotopy groups of $M$ are the groups $\pi^{*, *}\left(\mathcal{E}_{M}^{* *}, \bar{\partial}\right)$, which we denote $\pi^{*, *}(M)$. Note that $\mathcal{E}_{M}^{* ; *}$ as an augmented algebra depends on the base point. If $M$ has no nonconstant holomorphic functions, for example, if $M$ is compact, connected, then $H^{0,0}(M)=\mathrm{C}$ and it can be seen that $\pi^{*, *}(M)$ depends on the basepoint only up to (unnatural) isomorphism. In any case, we suppress the basepoint in our notation.

The de Rham cohomotopy groups of $M, \pi^{*}(M)$, are the groups $\pi^{*}\left(\mathcal{E}_{M}^{*}, d\right)$. We have Hurewicz maps $H^{p, q}(M) \rightarrow \pi^{p, q}(M)$ and $H^{p}(M) \rightarrow \pi^{p}(M)$. In $\S 5$, we will give both $\pi^{*, *}(M)$ and $\pi^{*}(M)$ the structure of graded Whitehead coalgebras, provided that $H^{0,0}(M)=\mathrm{C}$, respectively, $H^{0}(M)=\mathrm{C}$.

We now give three simple examples.

EXAMPLE. On p. 251 of [6], a minimal model for $\boldsymbol{P}^{n}$ (complex projective $\boldsymbol{n}$ space) is constructed. Their answer is $S(x, y)$, where $S$ denotes the free commutative algebra on $x$ and $y$ with $\operatorname{dim} x=2$ and $d y=x^{n+1}$. They work 
over the reals but it is clear that the complex valued de Rham forms have the same minimal model. Since $P^{n}$ is Kähler, $\bigoplus_{r} H^{p-r, r}\left(P^{n}\right) \cong H^{p}\left(P^{n}\right)$. Hence a minimal model (which is cofibrant) for the Dolbeault complex is $S(x, y)$ where $x$ has bidegree $(1,1)$ and $\bar{\partial} y=x^{n+1}$. From this it is easy to read off the complete structure of the Whitehead coalgebra.

EXAMPLE. The $2 n$ torus, $T^{2 n}$, is also Kähler. A minimal model is obtained by taking the exterior algebra on $2 n$ generators, $n$ of which have bidegree $(0,1)$ and $n$ of which have bidegree $(1,0) . \bar{\partial}$ is simply the zero map.

In both of these examples, the important facts are that the manifolds are Kähler and that their cohomology rings are particularly nice. Hence any other complex structure on these manifolds which is Kähler yields the same computations.

EXAMPLE. Complex $n$-space, $\mathbf{C}^{n}$, is Stein and $H^{*, *}\left(\mathbf{C}^{n}\right)=H^{*, 0}\left(\mathbf{C}^{n}\right)=$ the algebra of holomorphic forms. There is an inclusion $H^{*, 0}\left(\mathbf{C}^{n}\right) \rightarrow \mathcal{E}_{\mathbf{C}^{* * *}}^{*}$ and, in fact, $H^{*, 0}\left(C^{n}\right)$ with $\bar{\partial}=0$ is a cofibrant model. As an algebra over $H^{0,0}\left(C^{n}\right)=$ $\theta_{\mathbf{C}^{n}}=$ the ring of holomorphic functions, $H^{*, 0}\left(\mathbf{C}^{n}\right)$ is the exterior algebra generated by $d z_{1}, \ldots, d z_{n}$. If we choose the origin as basepoint, we find that $\pi^{0,0}$ and $\pi^{1,0}$ are the only nonzero Dolbeault cohomotopy groups and $\pi^{0,0}\left(C^{n}\right)$ $=\overline{\mathcal{O}}_{\mathbf{C}^{n}} / \overline{\mathcal{O}}_{\mathbf{C}^{n}}^{2}=$ the complex vector space generated by $z_{1}, \ldots, z_{n}$ with $\pi^{1,0}\left(\mathbf{C}^{n}\right)$ $=$ the complex vector space generated by $d z_{1}, \ldots, d z_{n}$.

The important fact about this last example is that $\mathbf{C}^{n}$ is Stein. In $\S 4$, we will provide a complete justification for this example.

It is possible to extend the above definitions to the relative case. To wit, let $M$ be a complex manifold and $A$ a closed analytic submanifold. The map $i^{*}$ : $\mathscr{E}^{*, *}(M) \rightarrow \mathcal{E}^{*, *}(A)$ is surjective and we set $\mathcal{E}^{*, *}(M, A)=\mathbf{C} \oplus \operatorname{ker} i^{*}$. $\mathcal{E}^{*}(M, A)$ is defined in a similar manner and it is an easy consequence of Sullivan's theory [6] that $\mathcal{E}^{*}(M, A)$ represents the "complex homotopy type" of $M / A$. We define $\pi^{*, *}(M / A)=\pi^{*, *}\left(\mathcal{E}^{*, *}(M, A)\right)$ and $\pi^{*}(M / A)=$ $\pi^{*}(\mathcal{E} *(M, A))$.

A DGA, DBA, or BBA, say $\mathcal{Q}$, is formal iff there exists a cofibrant algebra with maps (in our category) $\mathscr{B} \rightarrow \mathscr{Q}$ and $\mathscr{B} \rightarrow H^{*}(\mathbb{Q})$ (or $H^{*, *}(\mathbb{Q})$ ) which are weak equivalences. Cohomology is given the zero differential. Instead of saying that an algebra is formal, we sometimes say (following [6]) that the cohomotopy is a formal consequence of the cohomology.

A BBA, $\mathcal{Q}$, is $\partial$-degenerate iff there exists a BBA, $\mathfrak{N}$, such that

(1) the extra differential in $\mathscr{N}$ is identically zero,

(2) there is a map of BBA's $\Re \rightarrow \mathbb{Q}$,

(3) $\Re$ is a cofibrant model for $\mathscr{Q}$ as a DBA.

A BBA is strictly formal if it is $\partial$-degenerate and formal.

A manifold $M$ is de Rham formal iff $\mathcal{E}_{M}^{*}$ is a formal DGA. $M$ is Dolbeault formal iff $\mathcal{E}_{M}^{* * *}$ is formal, and $M$ is strictly formal iff $\mathcal{E}_{M}^{* * *}$ is strictly formal. It 
is easy to prove that strictly formal implies de Rham formal as well as Dolbeault formal.

In §3, we will compute minimal DBA models for Stein manifolds. Stein manifolds are Dolbeault formal, but not in general de Rham formal. The Calabi-Eckmann manifolds $M_{u, v}$ of $\$ 4$ are all Dolbeault formal, and the $M_{u, v}$ are strictly formal. The $M_{u, v}$ with $u>0$ are easily seen not to be $\partial$ degenerate.

An important class of examples of strictly formal manifolds are the compact Kähler manifolds, which we will discuss in $\$ 6$.

3. The Fröhlicher spectral sequence. A closed model category is given whenever specified classes of morphisms, called weak equivalences, fibrations, and cofibrations, respectively, satisfy the axioms CM2 to CM5 given by Quillen [17]. We make DGA's, DBA's, and homologically connected BBA's $\left(H^{0,0}=\mathrm{C}\right)$ into closed model categories by specifying that weak equivalences are $d, \bar{\partial}$, and $\bar{\partial}$ cohomology isomorphisms, respectively, fibrations are surjective maps, and cofibrations $A \rightarrow B$ are characterized by the lifting property

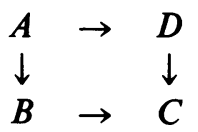

whenever $D \rightarrow C$ is a fibration and a weak equivalence. Notice that cofibrant DGA's, DBA's, or BBA's, $\mathbb{Q}$, defined in $\$ 2$, are exactly those $\mathbb{Q}$ such that $C \rightarrow Q$ is a cofibration.

Bousfield and Gugenheim [3] have shown that the category of DGA's is a closed model category. Exactly the same argument works for the category of DBA's and only minor variations are required to prove it for homologically connected BBA's. We leave this transcription as an exercise to the reader. However, we shall show below how to construct cofibrant models. This construction will illustrate why we restrict attention to homologically connected BBA's.

The natural functors $F$, which forgets the extra differential $\partial$ of a BBA to give a $\mathrm{DBA}$, and $A$, which amalgamates bidegree into total degree, give faithful embeddings of the two closed model categories, DBA's and homologically connected BBA's, into the closed model category of DGA's. By this, we mean that weak equivalences, fibrations, and cofibrations are all preserved. It follows from these observations that there are no results in $\$ \$ 4,5,6$, or 7 of [3], proved for DGA's, which do not remain valid for DBA's and homologically connected BBA's. Results in [3] (notably 6.4 and 6.13) prove that our definition of the cohomotopy of a DBA is well-defined and functorial. 
If $\mathcal{Q}$ is any DGA (respectively, DBA) concentrated in degree zero (respectively, total degree zero), then $Q$ is easily seen to be cofibrant. The analogous result is not true for BBA's (but $\mathrm{C}$ is always cofibrant, even when regarded as a BBA). It is for this reason that we restrict attention to homologically connected BBA's.

If $\mathcal{Q}$ is a DGA (DBA) and $x_{\alpha}$ is a set of generators with nonnegative degree (respectively, total degree), then an elementary extension of $\mathbb{Q}$ is $\mathscr{B}=\mathbb{Q} \otimes$ $\otimes_{\alpha} S\left(x_{\alpha}\right)$ where the differential on $\mathscr{B}$ extends that of $\mathbb{Q}$ and $d x_{\alpha} \in \mathbb{Q}$ (resp. $\left.\partial x_{\alpha} \in \mathbb{Q}\right)$. If $\mathbb{Q}$ is a BBA, this is modified to $\mathbb{Q} \otimes \otimes_{\alpha} S\left(x_{\alpha}, y_{\alpha}\right)$ where $\partial x_{\alpha}=y_{\alpha}$ and $\bar{\partial} x_{\alpha} \in \mathbb{Q}$. It is clear that an elementary extension of BBA's is an elementary extension of DBA's when we forget the $\partial$ differential.

If $x_{\alpha}$ is a set of cocycles in degree 0 in a DGA or DBA $\mathcal{Q}$, then $\mathscr{Q}$ modulo the ideal generated by the $x_{\alpha}$ 's is called an elementary contraction of $\mathscr{Q}$.

Bousfield and Gugenheim [3] show that, if $\mathscr{Q}$ is a cofibrant DGA, DBA, or BBA, any elementary extension of $\mathscr{Q}$ is cofibrant. If $\mathscr{Q}$ is a cofibrant DGA or DBA, then any elementary contraction of $\mathscr{Q}$ is cofibrant.

If $\mathcal{Q}$ is a homologically connected DGA (DBA, BBA), a cofibrant model for $\mathbb{Q}$ can be built by successive elementary extensions starting from $\mathbf{C}$. If $\mathbb{Q}$ is any DGA (or DBA), a cofibrant model for $\mathcal{Q}$ can be constructed by successive elementary extensions and contractions starting from $H^{0}(\mathbb{Q})$ (resp. $\left.H^{0,0}(\mathcal{Q})\right)[3]$.

Our first theorem is the cohomotopy analogue of a spectral sequence of Fröhlicher [8].

THEOREM 1. Given $M$, a connected complex manifold with no nonconstant holomorphic functions, there exists a spectral sequence, natural with respect to analytic maps, whose $E_{1}$ term is $\pi^{p, q}(M)$; whose $d_{r}$ has type $(r, 1-r)$; and which abuts to $\pi^{p+q}(M)$.

Remarks. Actual convergence is a bit tricky since $\pi^{p+q}$, unlike $H^{p, q}$, can have arbitrarily small negative $q$ 's. But strong convergence is clearly the case if, in addition, $H^{0,1}=H^{1,0}=0$. In fact, it follows from Theorem 6 in $\$ 6$ that $H^{1,0}=0$ is sufficient for convergence. The Hurewicz map takes the cohomology Fröhlicher spectral sequence to the cohomotopy analogue. We tend to refer to both of these spectral sequences as the Fröhlicher spectral sequence. In both spectral sequences, $d_{1}$ is induced by $\partial$. The cohomotopy spectral sequence is a spectral sequence of Whitehead coalgebras. (See \$6.)

Proof. Choose a cofibrant BBA model $\mathscr{B}$ for $\mathscr{E}_{M}^{* * *}$. This automatically a DBA model when we forget the $\partial$ differential. The hypothesis of no nonconstant holomorphic functions is that $H^{\mathbf{0 , 0}}(M)=\mathrm{C}$.

Following Fröhlicher we define a filtration on $\mathscr{B}$ whose $k$ th term contains all elements in $\mathscr{B}^{p, q}$ with $p \geqslant k$. This induces a filtration on $Q(\mathscr{B})$. These filtrations on $\mathscr{B}$ and $Q(\mathscr{B})$ are compatible with the differential $d=\partial+\bar{\partial}$ on 
$\Re$ and the induced differential on $Q(\Re)$, respectively.

The map from $\mathscr{B}$ to $\mathcal{E}_{M}^{* * *}$ maps the spectral sequence for $\mathscr{B}$ to the classical Fröhlicher spectral sequence. Since $\mathscr{B}$ is a DBA model, this map is an isomorphism on the $E_{1}$ terms. Hence, $H^{*}(\mathscr{B}, d) \rightarrow H^{*}(M)$ is an isomorphism and $\mathscr{B}$ is a DGA model for $\mathcal{E}_{M}^{*}$.

It follows that $H^{*}(Q(\mathscr{B}), d)$ is the de Rham cohomotopy. The spectral sequence coming from the filtration on $Q(\mathscr{B})$ abuts to this and its $E_{1}$ term is the Dolbeault cohomotopy. Naturality of this spectral sequence follows from homotopy naturality [3] of the choice of $\mathscr{B}$. The compatibility with the Hurewicz map comes from the map $\mathscr{B} \rightarrow Q(\mathscr{B})$.

REMARK. In \$4, we show, with the example of Stein manifolds, that there is no possibility of a cohomotopy Fröhlicher spectral sequence without the hypothesis of no nonconstant holomorphic functions.

RemarK. It is obvious that a $\partial$-degenerate manifold has $E_{1}=E_{\infty}$ in both the cohomology and cohomotopy Fröhlicher spectral sequences.

Theorem 1 admits a generalization. We say that $\mathscr{Q}$ is a filtered DGA if there is a decreasing sequence of differential submodules $F^{n} \mathscr{Q}$ with $F^{0} \mathscr{Q}=$ $A, \cap_{n=0}^{\infty} F^{n} \mathscr{Q}=0$, and $F^{n} \mathscr{Q} \cdot F^{m} \mathscr{Q}$ contained in $F^{n+m} \mathscr{Q}$.

Proposition 1. Let $\mathbb{Q}$ be a filtered DGA with a filtration that is finite in each degree. If $E_{0} \mathfrak{Q}$ is the associated $D B A$ and if $H^{0,0}\left(E_{0} \mathscr{Q}\right)=\mathrm{C}$, then there is a spectral sequence whose $E_{1}$ term is $\pi^{*, *}\left(E_{0} \mathscr{Q}\right)$ and whose abutment is $\pi^{*}(\mathfrak{Q})$. If in addition, $H^{1,0}=H^{0,1}=0$, then this spectral sequence converges.

Proof. We shall construct a filtered DGA $\mathscr{B}$ and a map $\mathscr{B} \rightarrow \mathbb{Q}$ of filtered DGA's such that $E_{0} \mathscr{B}$ is a cofibrant DBA model for $E_{0} \mathscr{Q}$. After this, the rest of the proof is identical to the proof of Theorem 1 and will be omitted. The filtration on $Q(\Re)$ induces the spectral sequence.

The construction of $\mathscr{B}$ goes as follows. Suppose we have constructed a cofibrant filtered DGA, $\mathscr{B}^{(p, q)}$, and a map $\mathscr{B}^{(p, q)} \rightarrow \mathbb{Q}$ such that $H^{r, s}\left(E_{0} \mathfrak{B}^{(p, q)}\right) \rightarrow H^{r, s}\left(E_{0} \mathfrak{Q}\right)$ is an isomorphism for all $r+s<p+q$ and $r+s=p+q, r<p$, and a monomorphism for $(r, s)=(p, q)$. Let $x_{\alpha}$ be a set of elements in $F^{p} Q^{p+q}$ which represent a basis for coker $H^{p, q} \rightarrow H^{p, q}$. Let $y_{\alpha}, z_{\alpha}$ be elements in one-to-one correspondence with $x_{\alpha}$ where degree $y_{\alpha}=p$ $+q$, degree $z_{\alpha}=p+q-1$ and filtration $y_{\alpha}=p$, filtration $z_{\alpha}=p+1$. Let $d y_{\alpha}=z_{\alpha}$ and form an elementary extension $\mathscr{B}_{1}^{(p, q)}=\mathscr{B}^{(p, q)} \otimes S\left(y_{\alpha}, z_{\alpha}\right)$. If we multiplicatively extend the filtration to $\mathscr{B}_{1}^{(p, q)}$, then there is an extension to a filtration preserving map $\mathscr{B}_{1}^{(p, q)} \rightarrow \mathbb{Q}$ with $y_{\alpha} \rightarrow x_{\alpha}, z_{\alpha} \rightarrow d x_{\alpha}$.

Now let $a_{\beta}$ be a set of representatives for a basis of ker $H^{p+1, q-1}\left(E_{0} \beta_{1}^{(p, q)}\right)$ $\rightarrow H^{p+1, q-1}\left(E_{0} \mathscr{Q}\right)$. Let $b_{\beta}, c_{\beta}$ be elements in one-to-one correspondence with $a_{\beta}$ where degree $b_{\beta}=p+q+1$, degree $c_{\beta}=p+q$ and filtration $b_{\beta}=p+$ 2 , filtration $c_{\beta}=p+1$. Let $d b_{\beta}=d a_{\beta}, d c_{\beta}=a_{\beta}-b_{\beta}$ and form $\mathfrak{B}^{p+1, q-1}=$ 
$\mathscr{B}_{1}^{(p, q)} \otimes S\left(b_{\beta}, c_{\beta}\right)$ with the obvious filtration. If we map $c_{\beta} \rightarrow \gamma_{\beta}$ and $b_{\beta} \rightarrow$ (image $\alpha_{\beta}$ ) $-d \gamma_{\beta}$, where (image $a_{\beta}$ ) $\equiv d \gamma_{\beta}$ modulo $F^{p+2} Q$, we get a filtration preserving extension to $\mathscr{B}^{p+1, q-1} \rightarrow \mathbb{Q}$.

We set

$$
\mathscr{B}^{-1, n+2}=\lim _{\rightarrow} \mathfrak{B}^{p, n-p} \quad \text { and } \mathfrak{B}^{\prime}=\lim _{\rightarrow} \mathfrak{B}^{-1, n} .
$$

Then $\mathscr{B} \rightarrow \mathbb{Q}$ is the required model. If the filtration is finite in each degree and $H^{1,0}=H^{0,1}=0$, then $\mathscr{B}$ has a filtration which is finite in each degree. Clearly, the spectral sequence will converge.

Remark. The spectral sequence in Proposition 1 is a spectral sequence of Whitehead coalgebras. (See \$6.)

4. The cohomotopy of Stein manifolds. In this section, we will compute the Dolbeault cohomotopy groups $\pi^{*, *}(M)$ when $M$ is a Stein manifold of complex dimension $n$.

THEOREM 2. If $M$ is a Stein manifold, then a cofibrant DBA model for $\mathcal{E}_{M}^{* * *}$ is given by $H^{*, 0}(M)$, the algebra of holomorphic forms, with $\bar{\partial}=0$.

Proof. By Cartan's Theorem B, [10], the inclusion $H^{*, 0}(M) \rightarrow \mathcal{E}_{M}^{* * *}$ is a weak equivalence. Hence, it suffices to show that $H^{*, 0}$ is a cofibrant DBA.

Since $M$ is Stein, $M$ can be analytically embedded as a closed submanifold of $C^{2 n+1}$ [10]. Hence, the cotangent bundle $\tau$ of $M$ has an analytic complement $\nu$.

It follows that $H^{1,0}(M)$, the sections of $\tau$, is a projective $H^{0,0}(M)$ module. In fact, $H^{*, 0}(M)$ is the exterior algebra, over the ring $H^{0,0}(M)$, on the module $H^{1,0}(M)$. To see this, notice that $H^{*, 0}(M)=\Gamma\left(\Lambda^{*} \tau\right)$, the space of sections of the exterior powers of $\tau$. But $\Gamma\left(\Lambda^{*} \tau\right)$ is a retract of

$$
\Gamma\left(\Lambda^{*}(\tau \oplus \nu)\right)=\Lambda_{H^{0.0}(M)}\left[d z_{1}, \ldots, d z_{2 n+1}\right],
$$

the exterior algebra generated by the restrictions of the canonical one forms. It follows that $H^{*, 0}(M)$ is an exterior algebra on $H^{1,0}(M)$ and also that $H^{*, 0}(M)$ is cofibrant, since it is a retract of a cofibrant DBA.

We can now compute the groups $\pi^{*, *}(M)$ when $M$ is a Stein manifold. Pick a point $p$ in $M$ and let $\bar{\theta}=\bar{H}^{0,0}(M)$, the ideal of holomorphic functions which vanish at $p$. Then $\pi^{0,0}(M)=\bar{\theta} / \bar{\theta} \cdot \bar{\theta}$ and $\pi^{1,0}(M)=H^{1,0}(M) / \bar{\theta}$. $H^{1,0}(M)$. All other Dolbeault cohomotopy groups of $M$ are zero!

Proposition 2. If $M$ is a Stein manifold of dimension $n$, then $\pi^{0,0}(M)$ and $\pi^{1,0}(M)$ are $n$ dimensional complex vector spaces.

Proof. $M$ can be embedded as a closed analytic subvariety of some $\mathbf{C}^{n+k}$ and we can assume that 0 is the basepoint in $M$ and $d z_{1}, \ldots, d z_{n}$ span the cotangent bundle of $M$ at 0 . We can find holomorphic functions $f_{1}, \ldots, f_{k}$ defined on $\mathbf{C}^{n+k}$ and vanishing on all of $M$ such that $f_{1}=\cdots=f_{k}=0$ are 
defining equations for $M$ in a neighborhood of 0 [10]. If we replace $f_{1}, \ldots, f_{k}$ by suitable linear combinations, we can assume that $d f_{i}=d z_{n+i}$ at 0 . Hence, $z_{i}=f_{i}+$ higher order terms.

If $f$ is any holomorphic function on $M$, then $f$ extends to a holomorphic function on $\mathbf{C}^{n+k}$, which we also denote by $f$. If $f$ vanishes at 0 , then $f=\sum_{i=1}^{n+k} a_{i} z_{i}+$ higher order terms. On $M, f=\sum_{i=1}^{n} a_{i} z_{i}+$ higher order terms. Hence $\pi^{0,0}(M)$ is $n$ dimensional with a basis represented by the local coordinates $z_{1}, \ldots, z_{n}$.

Likewise, $\pi^{1,0}(M)$ is $n$ dimensional with a basis represented by $d z_{1}, \ldots, d z_{n}$.

The above result shows that the connectivity assumption, $H^{0,0}=\mathrm{C}$, is necessary for the existence of a cohomotopy Fröhlicher spectral sequence, $\pi^{p, q} \Rightarrow \pi^{p+q}$. For example, any noncompact Riemann surface is Stein [10]. Even so simple a space as the punctured complex plane is a counterexample to the existence of the spectral sequence.

To show just how bad the situation is when $M$ is Stein, we observe that: For any finite simplicial complex $K$, there is a Stein manifold $M$ with the homotopy type of $K$. First, notice that, if $K$ has dimension $m, K$ can be embedded in $\Re^{2 m+1}$. Let $N^{2 m+1}$ be an open tubular neighborhood of $K$. Then $N$ is a real analytic manifold with the same homotopy type as $K$. A theorem of Grauert [9] says that $N$ can be embedded in a Stein manifold $M$ so that $M$ is a tubular neighborhood of $N$. ( $M$ is the complexification of $N$.) $M$ has the same homotopy type as $N$, hence as $K$.

When $M$ is Stein, $H^{*, *}(M)=H^{*, 0}(M)$ is the cofibrant DBA model, with $\bar{\partial}=0$. Hence, a Stein manifold is always Dolbeault formal but not always de Rham formal.

We close this section with the amusing observation that the de Rham homotopy type [6] of a Stein manifold is a formal consequence of the ring $H^{0,0}$ and the map $\partial: H^{0,0} \rightarrow H^{1,0}$.

5. The Eilenberg-Moore spectral sequence and the long exact homotopy sequence. We shall construct the analogue of the usual Eilenberg-Moore spectral sequence for Dolbeault cohomology. Borel [1] has constructed a Serre spectral sequence for the Dolbeault cohomology of an analytic fibre bundle with compact fibre, subject to the condition that the bundle be orientable in the sense given below. We will rely heavily on this spectral sequence in the proofs of Theorems 3 and 4.

Defintion. A complex analytic fibre bundle $(E, B, F, \pi)$ is said to be orientable if

(1) the action of the structure group of the bundle is locally constant on $H^{*, *}(F)$, and 
(2) the action of $\pi_{1}(B)$ is trivial on $H^{*, *}(F)$.

We recall [1] that any analytic fibre bundle with $\pi_{1}(B)=0$ and $F$ compact Kähler is orientable.

THEOREM 3. Let $(E, B, F, \pi)$ be an orientable analytic fibre bundle whose fibre is compact connected. Let $B_{1} \rightarrow B$ be an analytic map and consider the induced analytic fibre bundle $\left(E_{1}, B_{1}, F, \pi_{1}\right)$. There exists a spectral sequence of commutative algebras satisfying:

(1) the $E_{2}$ term is $\operatorname{Tor}_{H^{r}, q_{(B)}}\left(H^{*, *}\left(B_{1}\right), H^{*, *}(E)\right)$,

(2) the differential $d_{s}$ is of type $(s, 0,1-s)$,

(3) the $E_{\infty}$ term is the associated graded object to some decreasing filtration on $H^{*, *}\left(E_{1}\right)$ so that $H^{p, q}\left(E_{1}\right)$ has quotients $E_{\infty}^{r, p, q-r}$,

(4) the natural map from $H^{*, *}\left(B_{1}\right) \otimes_{H^{*, *}(B)} H^{*, *}(E)$ to $H^{*, *}\left(E_{1}\right)$ is given at the $E_{2}$ term by the edge homomorphism to Tor ${ }^{0, *, *}$.

Notice that the above spectral sequence is a second octant spectral sequence. Fixing $p$ may give total degree lines which are unbounded.

Proof. Given DBA's $\mathbb{Q}_{1}, \mathbb{Q}_{2}, \mathbb{Q}_{3}$ with maps $\mathbb{Q}_{2} \rightarrow \mathbb{Q}_{1}$ and $\mathbb{Q}_{2} \rightarrow \mathbb{Q}_{3}$, let $B\left(Q_{1}, \mathbb{Q}_{2}, \mathbb{Q}_{3}\right)$ denote the two-sided bar construction defined, for example, in [14]. Since $\mathbb{Q}_{1}, \mathbb{Q}_{2}, \mathbb{Q}_{3}$ are commutative, $B\left(\mathbb{Q}_{1}, \mathbb{Q}_{2}, \mathbb{Q}_{3}\right)$ has the structure of a DBA except for the fact that it may have nonzero elements in negative degrees. We will still call it a DBA. There is a natural map of DBA's from $B\left(\mathbb{Q}_{1}, \mathbb{Q}_{2}, \mathbb{Q}_{3}\right)$ to $\mathbb{Q}_{1} \otimes_{\mathbb{Q}_{2}} \mathbb{Q}_{3},[13, \S 12$ of Chapter $\mathrm{X}]$.

Denote $\mathcal{E}_{E}^{*, *}$ by $\mathcal{E}_{,} \mathcal{E}_{B}^{* * *}$ by $\mathscr{B}$, and $\mathcal{E}_{B_{1}}^{* * *}$ by $\Re_{1}$. There are maps $\mathscr{B} \rightarrow \mathcal{E}$ and $\mathscr{B} \rightarrow \mathscr{B}_{1}$. Form $B\left(\mathscr{G}_{1}, \mathscr{B}, \mathcal{E}\right)$ and consider the composition $B\left(\mathscr{B}_{1}, \mathscr{B}, \mathcal{E}\right) \rightarrow \mathscr{B}_{1} \otimes_{\mathscr{B}} \mathcal{E} \rightarrow \mathcal{E}_{E_{1}}^{* * *}$. Our task is to prove this composition a weak equivalence. Once we have done this, we have that $\operatorname{Tor}_{\mathscr{B}}\left(\mathscr{B}_{1}, \mathcal{E}\right) \rightarrow$ $H^{*, *}\left(E_{1}\right)$ is an isomorphism of algebras. The spectral sequence of our theorem is the bigraded version of the algebraic Eilenberg-Moore spectral sequence (Theorem 1.2 in [19]) constructed from the filtration of the bar construction by homological degree. Details of this may be found in [19].

Consider the Borel filtration [1] on $\mathcal{E}$ and $\mathcal{E}_{E_{1}}^{* * *}$. The $E_{1}$ terms of the resulting spectral sequence are $\mathscr{B} \otimes H^{*, *}(F)$ and $\mathscr{B}_{1} \otimes H^{*, *}(F)$, respectively [1, Lemma 5.2]. The $d_{1}$ differential on $1 \otimes H^{* * *}(F)$ is zero in both cases.

Recall that, as algebras, $B\left(\mathscr{B}_{1}, \mathscr{B}_{\mathcal{E}} \mathcal{E}\right)=B\left(\mathscr{B}_{1}, \mathscr{B}_{1} \mathrm{C}\right) \otimes \mathcal{E}$. Filter $B\left(\mathscr{B}_{1}, \mathscr{B}, \mathcal{E}\right)$ by using the tensor product filtration where we filter $\mathcal{E}$ by the Borel filtration [1] and we filter $B\left(\mathscr{B}_{1}, \mathscr{B}, C\right)$ by total degree. Note that this is a differential filtration, and that the DBA map $B\left(\mathscr{B}_{1}, \mathscr{B}, \mathcal{E}\right) \rightarrow \mathcal{E}_{E_{1}}^{* * *}$ is filtration preserving.

This filtration is the dual of that considered in [12]. The analogue of [12, (I.6.6)] is that 


$$
\begin{aligned}
E_{1}\left(B\left(\mathscr{B}_{1}, \mathscr{B}, \mathcal{E}\right)\right) & \cong B\left(\mathscr{B}_{1}, \mathscr{B}, E_{1}(\mathscr{E})\right) \\
& \cong B\left(\left(\mathscr{B}_{1}, \mathscr{B}, \mathscr{B}\right) \otimes H^{*, *}(F)\right) \\
& \cong B\left(\mathscr{B}_{1}, \mathscr{B}, \mathscr{B}\right) \otimes H^{*, *}(F)
\end{aligned}
$$

because of the structure of $d_{1} . E_{1}\left(B\left(\mathscr{B}_{1}, \mathscr{B}_{B}, \mathcal{E}\right)\right)$ maps to $\mathscr{B}_{1} \otimes_{\mathrm{C}} H^{*, *}(F)$ using the natural map $B\left(\mathscr{B}_{1}, \mathscr{B}, \mathscr{B}_{)} \rightarrow \mathscr{B}_{1} . \mathscr{B}_{1} \otimes_{\mathrm{C}} H^{*, *}(F) \cong E_{1}\left(\mathcal{E}_{E_{1}}^{* * *}\right)\right.$ and the map above is the map induced by $B\left(\mathscr{B}_{1}, \mathscr{B}, \mathcal{E}\right) \rightarrow \mathcal{E}_{E_{1}}^{* * *}$ on the $E_{1}$ terms.

Since $B\left(\mathscr{B}_{1}, \mathscr{B}, \mathscr{B}\right) \rightarrow \mathscr{B}_{1}$ is a weak equivalence,

$$
E_{2}\left(B\left(\mathscr{B}_{1}, \mathscr{B}, \mathcal{E}\right)\right) \rightarrow H^{*, *}\left(\mathscr{B}_{1}\right) \otimes H^{*, *}(F) \cong E_{2}\left(\mathcal{E}_{E_{1}}^{* * *}\right)
$$

is an isomorphism. By the standard comparison theorem [5] the map $B\left(\mathscr{B}_{1}, \mathscr{B}, \mathscr{E}\right) \rightarrow \mathcal{E}_{E_{1}}^{* * *}$ is a weak equivalence.

We remark that the spectral sequence in Theorem 3 has more structure than heretofore indicated. There is another differential, $\partial$ of type $(1,0)$, in the Dolbeault complex. $\partial$ induces another internal differential in all the above two-sided bar constructions. Hence, $\partial$ induces a filtration preserving derivation of type $(1,0)$ on $H^{*, *}\left(E_{1}\right)$ and a derivation of type $(0,1,0)$ on $\operatorname{Tor}_{\mathrm{H}^{* * *(B)}}\left(H^{*, *}\left(B_{1}\right), H^{*, *}(E)\right)$. In fact, $\partial$ induces a derivation $\partial$ of type $(0,1,0)$ on each $E_{s}$ term of the spectral sequence and $d_{s} \partial=-\partial d_{s}$.

It is clear from Borel [1] that $\partial$ induces a derivation throughout the Serre spectral sequence for $(E, B, F, \pi)$.

Both the Serre and the Eilenberg-Moore spectral sequences have antiholomorphic versions.

COROLlary 1. Under the hypotheses of Theorem 3, we have a spectral sequence of commutative algebras abutting to $H^{0, r+q}\left(E_{1}\right)$ whose $E_{2}$ term is $\operatorname{Tor}_{H^{r, *},(B)}^{r, q^{0, *}}\left(H^{0, *}\left(B_{1}\right), H^{0, *}(E)\right)$. There is also a spectral sequence converging to $H^{0, p+q}(E)$ whose $E_{2}$ term is $H^{0, p}(B) \otimes H^{0, q}(F)$.

Proof. The existence of the second spectral sequence is clearly in Borel [1]. The existence of the first is clear if we show that

$$
\operatorname{Tor}_{H^{\prime, * *(B)}}^{q, q}\left(H^{0, *}\left(B_{1}\right), H^{0, *}(E)\right) \rightarrow \operatorname{Tor}_{H^{* * *},(B)}^{r, q}\left(H^{*, *}\left(B_{1}\right), H^{*, *}(E)\right)
$$

is an isomorphism. But $H^{0, *}$ is a natural algebra retract of $H^{*, *}$, hence it follows.

In fact $\mathcal{E}^{0, *}$ is a differential algebra retract of $\mathcal{E}^{*, *}$. Another proof of the antiholomorphic Eilenberg-Moore spectral sequence can be given by following the proof of Theorem 3 and using the antiholomorphic Serre spectral sequence.

We next take up the Dolbeault analogue of the classical long exact homotopy sequence for a fibration.

THEOREM 4. Let $(E, B, F, \pi)$ be an orientable analytic fibre bundle whose fibre is compact connected and whose base has no nonconstant holomorphic 
functions. Then, for any $p \geqslant 0$, there is a long exact sequence

$$
\cdots \pi^{p, q-1}(F) \rightarrow \pi^{p, q}(B) \rightarrow \pi^{p, q}(E) \rightarrow \pi^{p, q}(F) \rightarrow \cdots .
$$

Proof. Denote $\mathcal{E}_{E}^{*, *}$ by $\mathcal{E}, \mathcal{E}_{B}^{*, *}$ by $\mathscr{B}$, and $\mathcal{E}_{F}^{*, *}$ by $\mathscr{F}$. Using $\S 3$ (and Quillen's CM5, [17]), choose cofibrant models $\mathscr{B}_{1}$ for $\mathscr{B}$ and $\mathcal{E}_{1}$ for $\mathcal{E}$ so that there is a commutative diagram

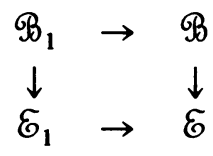

with $\mathcal{E}_{1} \rightarrow \mathcal{E}$ a fibration and $\mathscr{B}_{1} \rightarrow \mathcal{E}_{1}$ a cofibration. Since the fibration is orientable, we can assume $\mathcal{E}_{1}^{0}=\mathscr{B}_{1}^{0}=\mathbf{C}$. This is a simple consequence of Borel's Serre spectral sequence. Then $\mathscr{F}_{1}=\mathbf{C} \otimes_{\mathscr{B}_{1}} \mathscr{E}_{1}$ is cofibrant since pushouts of cofibrations are cofibrations.

Consider the natural map $\mathscr{F}_{1} \rightarrow \mathscr{F}$. We wish to prove that this is a weak equivalence, hence that $\mathscr{F}_{1}$ is a cofibrant model for $\mathscr{F}$. If $\mathscr{B}_{1}$ were connected and zero in total degree one, then $\mathscr{B}_{1} \rightarrow \mathcal{E}_{1} \rightarrow \mathscr{F}_{1}$ would automatically be an orientable "algebraic fibration." The result would then follow from the Moore comparison theorem [5]. But, in general, this is not so, and we have to use the proof of Theorem 3.

By the proof of Theorem 3, there is a commutative diagram

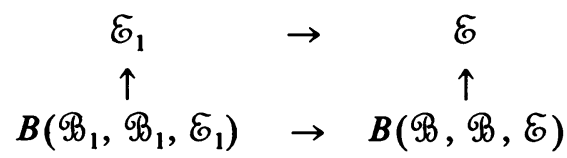

in which the vertical maps and the top map are weak equivalences. Hence, so is the bottom map. Since $B\left(\mathscr{B}_{1}, \mathscr{B}_{1}, \mathcal{E}_{1}\right) \rightarrow \mathcal{E}_{1}$ is a surjective weak equivalence and $\mathscr{E}_{1}$ is cofibrant, we can find a splitting (by Quillen's axiom CM4, [17]) so that

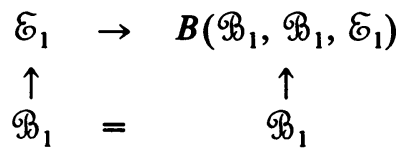

commutes.

REMARK. Even though $B\left(\mathscr{B}_{1}, \mathscr{B}_{1}, \mathscr{E}_{1}\right)$ is an algebra with nonzero negative degrees, this argument is valid since $\mathscr{E}_{1}=\mathbf{C}$.

There is an induced commutative diagram

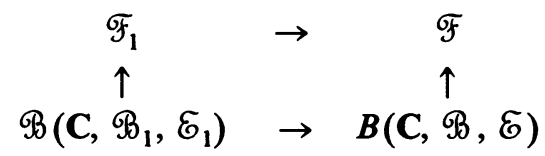

and a splitting $\mathscr{F}_{1} \rightarrow \mathscr{B}\left(C, \mathscr{B}_{1}, \mathscr{E}_{1}\right)$. In this diagram, the vertical maps are weak equivalences by the proof of Theorem 3 . That the bottom map is a weak 
equivalence is a standard argument using the algebraic Eilenberg-Moore spectral sequence [19, Theorem 1.2], and using Theorem 7.6 of [7]. To apply 7.6 involves checking that certain completeness hypotheses are satisfied. We leave this to the reader.

But the above diagram, together with the splitting, shows that $\mathscr{F}_{1} \rightarrow \mathscr{F}$ is a weak equivalence.

Now, $\mathscr{B}_{1}, \mathscr{E}_{1}$, and $\mathscr{F}_{1}$ are cofibrant models and we have a short exact sequence

$$
0 \rightarrow Q\left(\mathscr{P}_{1}\right) \rightarrow Q\left(\mathcal{E}_{1}\right) \rightarrow Q\left(\mathscr{F}_{1}\right) \rightarrow 0
$$

by $[3,6.15]$. The resulting long exact cohomology sequence is the sequence we desire.

We have shown that the long exact cohomotopy sequence exists. We now wish to show that the connecting homomorphism is well-defined and natural.

To see this, suppose that $\Re \rightarrow \Re \rightarrow \mathscr{P}$ is a sequence of cofibrant DBA's with a short exact sequence of indecomposables, and suppose that there is a homotopy commutative diagram

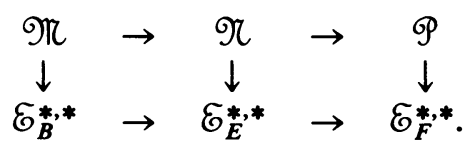

Since $\mathscr{T}, \mathfrak{T}$, and $\mathscr{P}$ are cofibrant, we can find maps

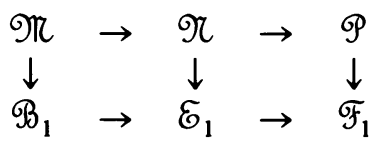

making a homotopy commutative diagram. (See $[3,6.4]$.) Hence

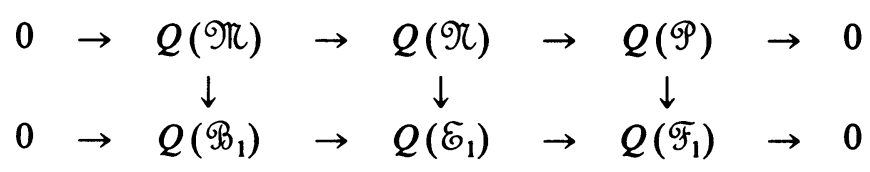

commutes up to chain homotopy. That the connecting homomorphism is well-defined and natural is now standard.

REMARK. If the base $B$ in Theorem 4 has no nonconstant holomorphic functions, then it follows from Borel's Serre spectral sequence that $E$ has no nonconstant holomorphic functions. Hence, the Fröhlicher spectral sequences of $F, E, B$ are all defined. Under these conditions, the connecting homomorphism $\pi^{p, q-1}(F) \rightarrow \pi^{p, q}(B)$ induces a morphism of type $(0,1)$ from the Fröhlicher spectral sequence of $F$ to that of $B$. The induced map on the $E_{\infty}$ terms is the associated graded of the usual map $\pi^{p+q-1}(F) \rightarrow \pi^{p+q}(B)$, which does preserve the Fröhlicher filtration. This can be proved by repeating the proof of Theorem 4 using BBA models. One obtains a short exact sequence of indecomposables with the structure of a $\partial$ and a $\bar{\partial}$ differential. 
Replace the indecomposables of the model for the base by the standard mapping cone construction on the map from the indecomposables of the total space model to those of the fibre model. One obtains the connecting homomorphism on the chain level as a morphism of filtered objects. A morphism of spectral sequences is induced.

As examples of the above theory, we compute our invariants for the Calabi-Eckmann manifolds. Let $\boldsymbol{M}_{u, v}$ denote any of the complex manifolds diffeomorphic to $S^{2 u+1} \times S^{2 v+1}$ constructed by Calabi and Eckmann [4]. $M_{u, v}$ is the total space of an analytic fibre bundle with fibre a torus and base $P^{u} \times P^{v}$. By the remark after our definition of orientable, this bundle is orientable. $M_{u, v}$ is also the total space of an analytic fibre bundle with fibre $M_{0, v}$ and base $P^{v}$, where the complex structure on $M_{0, v}$ depends on the one on $M_{u, v}$. By Borel, [1 (9.3)], this bundle is also orientable.

Consider the bundle $T^{2} \rightarrow M_{0, v} \rightarrow P^{v}$ for $v>0$. Lemma 9.4 of Borel [1] says that $H^{1,0}\left(M_{0, v}\right)=0$, so by the holomorphic Hurewicz theorem (Corollary 2 in $\S 6) \pi^{1,0}\left(M_{0, v}\right)=0$. The coboundary map in the long exact sequence is now forced from our calculations of $\pi^{*, *}\left(T^{2}\right)$ and $\pi^{*, *}\left(P^{v}\right)$ in $\$ 2$. We have that $\pi^{1,0}\left(T^{2}\right)$ goes isomorphically to $\pi^{1,1}\left(P^{v}\right)$ and the reader can easily work out $\pi^{*, *}\left(M_{0, v}\right)$ from this.

Feeding our answer back into the long exact sequence for $M_{0, v} \rightarrow M_{u, v} \rightarrow$ $P^{u}$, we can calculate $\pi^{*, *}\left(M_{u, v}\right) \cdot M_{0,0}$ is just the torus, so assume $u+v>0$. Then $\pi^{p, q}\left(M_{u, v}\right)$ is given by

\begin{tabular}{c|c|c|c|c|c}
$(p, q)$ & $(0,1)$ & $(1,1)$ & $(u+1, u)$ & $(v+1, v)$ & any other \\
\hline$\pi^{p, q}$ & $\mathbf{C}$ & $\mathbf{C}$ & $\mathbf{C}$ & $\mathbf{C}$ & 0
\end{tabular}

if $u \cdot v>0$. If either $u$ or $v$ is 0 , the terms $(1,1)$ and $(0,1)$ drop out.

A cofibrant model is easily constructed. Choose generators $x_{0,1}, x_{1,1}, x_{u+1, u}$, and $x_{v+1, v}$ and form $S\left(x_{0,1}, x_{1,1}, x_{u+1, u}, x_{v+1, v}\right), \bar{\partial} x_{0,1}=\bar{\partial} x_{1,1}=0$. If $u<v$, $\bar{\partial} x_{u+1, u}=x_{1,1}^{u+1}$ as one can see from the analytic map $M_{u, v} \rightarrow P^{u}$ if $u>0$, and the relation is also needed if $u=0$. (If $v<u$, take $\bar{\partial} x_{v+1, v}=x_{1,1}^{v+1}$.) $\bar{\partial} x_{v+1, v}$ $=0\left(\bar{\partial} x_{u+1, u}=0\right)$. Hence, for $u \cdot v>0$ we have built a minimal model, but if $u \cdot v=0$, we have only built a cofibrant one.

From the model, one can read off Borel's calculation of the Dolbeault cohomology rings [1].

Define $\partial x_{0,1}=x_{1,1}, \partial x_{u+1, u}=\partial x_{v+1, v}=0$. This defines an extra differential in our model for $\mathcal{E}_{\mathcal{M}_{u, 0}^{* *}}^{* *}$ compatible with the $\partial$ there. The Fröhlicher spectral sequence for either cohomotopy or cohomology collapses at the $E_{2}$ term unless $u \cdot v=0$, whereupon the $E_{1}$ term is $E_{\infty}$.

It is easily seen that the $M_{u, v}$ are all Dolbeault formal. But only $M_{0, v}$ are d-degenerate. 
6. Whitehead coalgebras and the Hurewicz theorems. If $A$ is a graded or a bigraded vector space, define $\tau: A \otimes A \rightarrow A \otimes A$ by $\tau(a \otimes b)=(-1)^{|a||b|}$ where || denotes degree or total degree, respectively. Define $\sigma: A \otimes A \otimes A$ $\rightarrow A \otimes A \otimes A$ by $\sigma(a \otimes b \otimes c)=(-1)^{|a||b|+|a||c|} b \otimes c \otimes a$.

Recall that homotopy groups have a bilinear pairing, the Whitehead product. The dual notion is that of a Whitehead coalgebra.

Definition. A Whitehead coalgebra is a (bi)graded vector space $A$ and a linear cobracket operation $\mho: A \rightarrow A \otimes A$ of degree +1 (type $(0,1))$ which satisfies

(1) $(1-\tau) \mathcal{W} \equiv 0$,

(2) (the Jacobi identity). If $\omega: A \otimes A \rightarrow A \otimes A$ is defined by $\omega(a \otimes b)=$ $(-1)^{|a|} a \otimes b$, then $\left(1+\sigma+\sigma^{2}\right)(1 \otimes \mho) \omega W \equiv 0$.

(The reader can define a Whitehead algebra by reversing the above arrows.)

Following Sullivan [6], we give the cohomotopy of a homologically connected DGA, $Q$, with $H^{0} \mathscr{Q}=\mathrm{C}$, the structure of a Whitehead coalgebra. Choose a cofibrant model $\mathscr{B}$ for $\mathcal{Q}$. Recall that $\pi^{*}(\mathscr{Q}) \cong H^{*}(Q(\mathscr{B}))$ and, since $Q$ is homologically connected, we can assume that $\mathscr{B}$ is free commutative. We now give two definitions of the Whitehead cobracket $\mathscr{}$.

We can filter $\mathscr{B}$ by the powers of its augmentation ideal. $E_{1}^{1, *-1}$ of the resulting spectral sequence is $H^{*}(Q(\mathscr{B})) . E_{1}^{2, *-2}$ is the subset of $\bigoplus_{p} H^{p}(Q(\mathscr{B})) \otimes H^{*-p}(Q(\mathscr{B}))$ which is invariant under $\tau$. $\mathscr{W}$ is just the $d_{1}$ differential. From this definition, it is clear that $W$ is natural. That $W$ is actually a Whitehead cobracket will be checked from our second definition. We remark that the same procedure defines $W$ for the cohomotopy of a homologically connected DBA, $H^{0,0}=\mathrm{C}$.

For certain results a chain level definition of $W$ is desirable. Unfortunately this definition is not natural since we begin by choosing a map $S(Q(\Re)) \rightarrow$ $\mathscr{B}$, where $S(Q(\mathscr{B})$ ) denotes the free commutative algebra on $Q(\mathscr{B})$. This map is an isomorphism by $[3,7.11]$; hence, any element in $\mathscr{B}$ is expressible as a unique sum of an element from $\mathbf{C}$, plus an element from $Q(\Re)$, plus an element from $Q(\Re) \cdot Q(\Re)$, etc. Notice that $Q(\mathscr{B}) \cdot Q(\Re)$ is contained in $Q(\Re) \otimes Q(\mathscr{B})$ as the kernel of $1-\tau$. Define $\mathscr{W}: Q(\mathscr{B}) \rightarrow Q(\mathscr{B}) \otimes Q(\mathscr{B})$, on an element $a$, by $d a=d_{Q} a+W(a)+$ junk, where $d_{Q} a$ is in $Q(\Re)$, "junk" is in triple and higher products, and $\mathscr{W ( a )}$ is in $Q(\Re) \cdot Q(\Re)$.

The differential $Q(\mathscr{B}) \rightarrow Q(\mathscr{B})$ is just $a \rightarrow d_{Q} a$ and it is easy to check that $\left(d_{Q} \otimes 1+1 \otimes d_{Q}\right)$ W $(a)=-\mho\left(d_{Q} a\right)$. Hence, $\widetilde{\text { induces }} \mho: H^{*}(Q(B)) \rightarrow$ $H^{*}(Q(\Re)) \otimes H^{*}(Q(\Re))$. We leave to the reader the following points. First, that our two definitions agree. Second, that either definition makes $H^{*}(Q(\mathscr{B}))$ into a Whitehead coalgebra. Hint: The latter is most easily checked by applying the second definition to a $\mathscr{B}$ with decomposable 
differential (see $[3,7.7])$. The Jacobi identity follows by symmetrizing the relation $d^{2}=0$.

In $\S 3$, we mentioned that the Fröhlicher spectral sequence was a spectral sequence of Whitehead coalgebras. This is a trivial consequence of the fact that our second definition of the Whitehead cobracket preserves the Fröhlicher filtration on a BBA model. The spectral sequence in Proposition 1 of $\$ 3$ is a spectral sequence of Whitehead coalgebras for essentially the same reason.

Our next project is to prove four Hurewicz theorems. There is an "Hurewicz map" for any DBA, and we begin with

LEMMA 1. Let $\mathbb{Q}$ be any DBA such that $H^{0,0}(\mathscr{Q}) \cong \mathrm{C}$. Then $H^{1,0} \rightarrow \pi^{1,0}$ and $H^{0,1} \rightarrow \pi^{0,1}$ are isomorphisms onto the kernel of the Whitehead cobracket.

LEMMA 2. Let $\mathbb{Q}$ be a $D B A$ and suppose that $\bar{H}^{p, q}(\mathfrak{Q})=0$ for $p+q<t$, where $t \geqslant 0$. Then $H^{r, s}(Q) \rightarrow \pi^{r, s}(\mathbb{Q})$ is an isomorphism for $0<r+s<2 t-$ 1 , and $Q_{r, s}\left(H^{*, *}(\mathbb{Q})\right) \rightarrow \pi^{r, s}(\mathbb{Q})$ is a monomorphism for $r+s<3 t-2$. Further, we have a long exact sequence

$$
\begin{aligned}
\cdots & \rightarrow H^{r, s}(\mathbb{Q}) \rightarrow \pi^{r, s}(\mathbb{Q}) \rightarrow\left[\oplus H^{t, v}(\mathbb{Q}) \otimes H^{r-t, s-v+1}(\mathbb{Q})\right]^{Z_{2}} \\
& \rightarrow H^{r, s+1}(\mathbb{Q}) \rightarrow \cdots \rightarrow \pi^{r, 3 t-r-2}(\mathbb{Q}) .
\end{aligned}
$$

We explain our maps and our notation in the course of the proof.

Proofs. We begin to construct a cofibrant model for $\mathcal{Q}$. Lemma 1 is immediately obvious. By hypothesis in Lemma 2, the first generators are introduced in some dimension which has total degree at least $t$. In fact, it is easy to see that one need only introduce cycle generators in all dimensions through $2 t-1$. This proves the first part of Lemma 2.

In the long exact sequence, the map from $H^{r, s}$ to $\pi^{r, s}$ is just the Hurewicz map. [ ] $]^{Z_{2}}$ denotes the subgroup of the tensor product fixed under the canonical involution on a graded tensor product. The map from []$^{Z_{2}}$ to $H^{r, s+1}$ is just multiplication in the cohomology ring. The map from $\pi^{r, s}$ to []$^{Z_{2}}$ is given by the Whitehead cobracket followed by the inverse of the Hurewicz maps (which are isomorphisms by the first paragraph). The proof that the sequence is long exact is a standard consequence of the collapse of the defining spectral sequence for Whitehead cobrackets through the range $(r, 3 t-r-2)$.

We note that Lemmas 1 and 2 have obvious restatements in terms of $\pi^{p, q}(M)$ and $H^{p, q}(M)$ or $\pi^{p}(M)$ and $H^{p}(M)$. We now apply Lemma 2 to prove a more subtle Hurewicz theorem.

THEOREM 5. Given $M$, a connected complex manifold with no nonconstant holomorphic functions, suppose $H^{0, p}(M)=0$ for $0<p<t$ where $t \geqslant 0$. Then $H^{0, r}(M) \rightarrow \pi^{0, r}(M)$ is an isomorphism for $r<2 t-1$ and 


$$
\begin{aligned}
\cdots & \rightarrow H^{0, r}(M) \rightarrow \pi^{0, r}(M) \rightarrow\left[\oplus H^{0, s}(M) \otimes H^{0, r-s+1}(M)\right]^{Z_{2}} \\
& \rightarrow H^{0, r+1}(M) \rightarrow \cdots \rightarrow \pi^{0,3 t-2}(M)
\end{aligned}
$$

is exact.

Proof. Consider $\mathbb{Q}=\bigoplus \mathcal{E}^{0, p}$ for all $p \geqslant 0$. $\mathcal{Q}$ is clearly a differential subalgebra of $\left(\mathcal{E}_{M}^{* *}, \bar{\partial}\right)$. Moreover the projection $\mathcal{E}_{M}^{* * *} \rightarrow \mathbb{Q}$ is a map of differential algebras. Hence, $\pi^{0, *}(\mathscr{Q})$ is isomorphic to $\pi^{0, *}(M)$ and $H^{0, *}(\mathscr{Q})$ is isomorphic to $H^{0, *}(M)$. Theorem 4 follows from Lemma 2 applied to $\mathbb{Q}$.

We call Theorem 5 the antiholomorphic Hurewicz theorem. A weaker holomorphic Hurewicz theorem is also valid. To wit, we have a map of DBA's, $H^{*, 0} \rightarrow \mathcal{E}^{*, *}$, and hence a map of the "formal holomorphic fan," $\pi^{*, *}\left(H^{*, 0}(M)\right)$, into $\pi^{*, *}(M)$. We call $\pi^{*, *}\left(H^{*, 0}(M)\right)$ a formal fan since building a cofibrant model for $H^{*, 0}(M)$, unlike the problem for $H^{0, *}(M)$, always requires the introduction of cohomotopy with negative $q$, unless $H^{*, 0}(M)$ is an exterior algebra on odd dimensional generators. Define an integer $\psi(t)$ as follows. Let $c(j)$ be the smallest integer such that $H^{c(j) j}(M) \neq$ 0 . Let $t \leqslant c(0)$ and define $\psi(t)$ to be the minimum of $c(1)+t, c(2)+$ $2 t, \ldots, c(j)+j t, \cdots$.

THEOREM 6. If $H^{0,0}(M)=\mathrm{C}$ and $H^{p, 0}(M)=0$ for $0<p<t$, then the map $\pi^{p, q}\left(H^{*, 0}(M)\right) \rightarrow \pi^{p, q}(M)$ is:

(a) an isomorphism for $p<q t+\psi(t)$ for all $q \leqslant 0$,

(b) a monomorphism for $p<(1-q) t+\psi(t)$ for all $q \leqslant 0$.

ReMARKs. For $p<t+\psi(t)$, the Hurewicz image of $H^{p, 0}(M) \rightarrow \pi^{p, 0}(M)$ is the same as the Hurewicz image in the formal fan. By Theorem 12, this is just $Q H^{p, 0}(M)$. Two other consequences of Theorem 6 are:

If $H^{*, 0}(M)=0$, then $\pi^{p, q}(M)=0$ for $q<0$.

If $H^{1,0}(M)=0$, then total degree lines are bounded in the cohomotopy Fröhlicher spectral sequence and it converges.

Proof of Theorem 6. Build a minimal model $\Re^{*, *}$ for $\mathcal{E}_{M}^{* * *}$ and a minimal model $\mathbb{Q}^{*, *}$ for $H^{*, 0}(M)$. We have a map $\mathbb{Q} \rightarrow \mathscr{B}$.

Notice first that $\mathbb{Q}^{p, q}=\mathfrak{B}^{p, q}=0$ for $p<(1-q) t$. This is clear if $q>1$ since we never get any generators with negative $p$ 's. If $p+q<t$, then we need only add generators with $q \geqslant 1$. If $p+q=t$ then we must add some generators of type $(t, 0)$ but none with $q<0$. The first time we need generators with $q=-1$ is in total degree $2 t-1$. In general, the first time we need generators with $q=-k$ is in total degree $(1+k) t-k$. Hence $p>(1$ - q) $t$ before we get anything on the $q$-line if $q<0$.

Next we claim $\mathbb{Q}^{p, q} \rightarrow \mathscr{B}^{p, q}$ is an isomorphism if $p<-q t+\psi(t)$. Well, the map is an isomorphism until there is a decomposable in $\mathscr{B}^{p, q}$ which involves a $\mathscr{B}^{r, s}$ with $s>1$. Suppose our decomposable comes from $\pi^{x_{1}, i_{1}} \otimes \cdots \otimes \pi^{x_{r}, i_{r}}$. 
If $i_{j}>0$, then $x_{j} \geqslant \psi(t)-t i_{j}$ by the definition of $\psi(t)$, and if $i_{j}<0, x_{j} \geqslant(1$ $\left.-i_{j}\right) t$ by the last paragraph.

Hence

$$
p=\sum x_{j} \geqslant a \psi(t)+(r-a) t-t \sum i_{j} \equiv a \psi(t)+(r-a)-(q+1) t,
$$

where $a$ is the number of positive $i_{j}$ 's.

Since $a \geqslant 1$ by assumptions and $r \geqslant 2$ for the same reason, and since $\psi(t)$ is obviously at least $t$

$$
\begin{aligned}
p & \geqslant \psi(t)+(a-1) \psi(t)+(r-a) t-(q+1) t \\
& \geqslant \psi(t)+(a-1) t+(2-a) t-(q+1) t
\end{aligned}
$$

or

$$
p \geqslant-q t+\psi(t)
$$

which proves our claim.

This is also part (a) of our theorem. To see (b) consider the spectral sequence introduced in the first part of $\$ 6$. For simplicity of notation, we shall use the grading convention that $E_{r, p, q}^{s}$ has total degree $p+q$. Recall $E_{r, p, q}^{1}$ is the $r$-fold symmetric product of $\pi^{*, *}$.

Since $E_{1, p, q}^{1}(\mathscr{Q}) \rightarrow E_{1, p, q}^{1}(\mathscr{B})$ is an isomorphism for $p<-q t+\psi(t)$, estimates like those we used to show $\mathbb{Q}^{p, q}=0$ for $p<(1-q) t$ can be used to show $E_{r, p, q}^{1}(Q) \rightarrow E_{r, p, q}^{1}(\Re)$ is an isomorphism for $p<(r-1-q) t+\psi(t)$.

Suppose that somehow we have proved that $E_{r, q, q}^{1}(\mathscr{Q}) \rightarrow E_{r, p, q}^{1}(\mathscr{B})$ is a monomorphism for $p<(r-q) t+\psi(t)$ for all $q>q_{0}$. (If $q_{0}=0$ this is easy since $E_{r, p, q}^{1}(\mathbb{Q})=0$ if $q>0$.)

If we can use the above to show that $E_{r, p, q_{0}}^{1}(\mathscr{Q}) \rightarrow E_{r, p, q_{0}}^{1}(\Re)$ is a monomorphism for $p<\left(r-q_{0}\right) t+\psi(t)$ thus we have part (b) using induction and setting $r=1$.

Moreover, it is enough to use our inductive hypothesis to show $E_{1, p, q_{0}}^{1}(\mathbb{Q}) \rightarrow$ $E_{1, p, q_{0}}^{1}(\Re)$ is monic for $p<\left(1-q_{0}\right) t+\psi(t)$ for then we can use our estimates and our inductive hypothesis to show $E_{r, p, q_{0}}^{1}(\mathscr{Q}) \rightarrow E_{r, p, q_{0}}^{1}(\Re)$ is monic for $p<\left(r-q_{0}\right) t+\psi(t)$.

Now recall $d_{s}: E_{r, p, q}^{s} \rightarrow E_{r+s, p, q+1}^{s}$. We have $E_{r+1, p, q_{0}}^{1}(\mathbb{Q}) \rightarrow E_{r+1, p, q_{0}}^{1}(\mathscr{B})$ monic for $p<\left(r-q_{0}\right) t+\psi(t)$ and $E_{r, p, q_{0}}^{1}(\mathbb{Q}) \rightarrow E_{r, p, q_{0}}^{1}(\mathscr{B})$ iso. (hence epic) for $p<\left(r-1-\dot{q}_{0}\right) t+\psi(t)$.

Assume by induction that $E_{r+s, p, q_{0}+1}^{s}(\mathscr{Q}) \rightarrow E_{r+s, p, q_{0}+1}^{s}(\mathscr{B})$ is monic for $p<\left(r-q_{0}\right) t+\psi(t)$ and that $E_{r, p, q_{0}}^{s}(\mathscr{Q}) \rightarrow E_{r, p, q_{0}}^{s}(\mathscr{B})$ is epic for $p<(r-1-$ $\left.q_{0}\right) t+\psi(t)$.

It is now straightforward to show that, if $p<\left(r-1-q_{0}\right) t+\psi(t)$ then

$$
E_{r+s, q_{0}+1}^{s+1}(\mathscr{Q}) \rightarrow E_{r+s, p, q_{0}+1}^{s+1}(\mathscr{B}) \text { is monic }
$$


and

$$
E_{r, p, q_{0}}^{s+1}(\mathscr{Q}) \rightarrow S_{r, p, q_{0}}^{s+1}(\mathscr{B}) \text { is epic. }
$$

Hence

$$
E_{r+s+1, p, q_{0}+1}^{s+1}(\mathscr{Q}) \rightarrow E_{r+s+1, p, q_{0}+1}^{s+1}(\Re) \text { is monic for } p<\left(r-q_{0}\right) t+\psi(t) \text {. }
$$

Since $\mathscr{Q}$ and $\mathscr{B}$ are connected, the spectral sequences converge to $H^{p, q}$. We have that

$$
E_{r, p, q_{0}}^{\infty}(\mathscr{Q}) \rightarrow E_{r, p, q_{0}}^{\infty}(\mathscr{B}) \text { is epic for } p<\left(r-1-q_{0}\right) t+\psi(t) .
$$

Hence if $p<\left(1-q_{0}\right) t+\psi(t)$ and $q_{0}<0$, we have

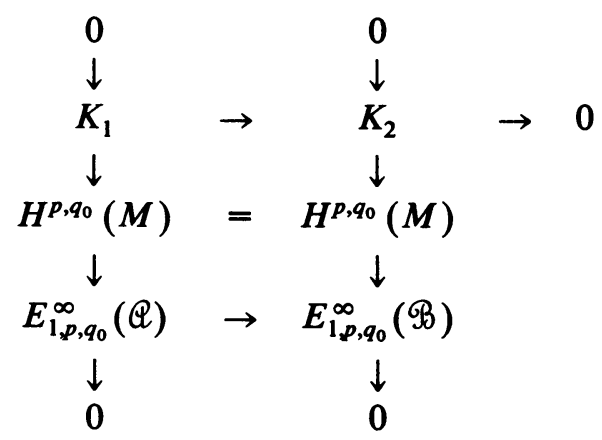

with exact columns.

That the map between the kernels $K_{1} \rightarrow K_{2}$ is epic is merely a reformulation of the fact that

$$
E_{r, p, q_{0}}^{\infty}(\mathscr{Q}) \rightarrow E_{r, p, q_{0}}^{\infty}(\mathscr{B}) \text { is epic for } r \geqslant 2
$$

since $p<\left(1-q_{0}\right) t+\psi(t) \leqslant\left(r-1-q_{0}\right) t+\psi(t)$ if $r \geqslant 2$.

Hence $E_{1, p, q_{0}}^{\infty}(\mathbb{Q}) \cong E_{1, p, q_{0}}^{\infty}(\mathscr{B})$ which is our claim about the Hurewicz homomorphism.

Now no differentials come into $E_{1, p, q_{0}}^{\infty}$ so $E_{1, p, q_{0}}^{1}$ is filtered by $E_{1, p, q_{0}}^{1} \supset E_{1, p, q_{0}}^{2}$ $\supset \cdots \supset E_{1, p, q_{0}}^{\infty}$. Suppose there is a nontrivial kernel in $E_{1, p, q_{0}}^{1}(\mathbb{Q}) \rightarrow$ $E_{1, p, q_{0}}^{1}(\Re)$ and suppose by induction that it has persisted to $E^{s} . d^{s}$ lands in $E_{s+1, p, q_{0}+1}^{s}$ and $E_{s+1, p, q_{0}+1}^{s}(\mathscr{Q}) \rightarrow E_{s+1, p, q_{0}+1}^{s}(\mathscr{B})$ is monic for $p<\left(1-q_{0}\right) t+$ $\psi(t)$. Hence any element in the kernel of $E_{1, p, q_{0}}^{s}(\mathscr{Q}) \rightarrow E_{1, p, q_{0}}^{s}(\mathscr{B})$ persists to $E^{\infty}$. Hence $E_{1, p, q_{0}}^{s}(\mathbb{Q}) \rightarrow E_{1, p, q_{0}}^{s}(\mathscr{B})$ is monic for $p<\left(1-q_{0}\right) t+\psi(t)$.

COROLlary 2. Given $M$, a connected complex manifold with no nonconstant holomorphic functions, suppose $H^{p, 0}(M)=0$ for $0<p<t$. Then $\pi^{p, 0}(M)=0$ for $0<p<t$ and $H^{r, 0}(M) \rightarrow \pi^{r, 0}(M)$ is an isomorphism onto the kernel of the Whitehead cobracket for $r<2 t$.

Proof. The first statement is a direct consequence of Theorem 6. To prove the second statement, use Theorem 6 and note that, if $r<2 t, \mathscr{B}^{r, 1}$ contains 
only indecomposables and second order decomposables, where $\mathscr{B}^{* * *}$ is a minimal model for $\mathcal{E}_{M}^{* * *}$.

REMARK. It is possible to prove Corollary 2 independently of Theorem 6. The proof is not difficult.

To state our next theorem, the "sweep Hurewicz theorem," we define functions $\delta(r)$ and $c(M)$. Given $r \geqslant 0$ define $\delta(r)$ to be the smallest integer $p+q$ with $q / p \geqslant r$ and with $H^{p, q}(M) \neq 0$. In other words $\delta(r)$ is the smallest total degree of the nonzero elements in the sector between the line of slope $r$ and the $y$-axis. Define $c(M)$ to be $p+q-1$ where $p+q$ is the smallest positive integer such that $H^{p, q}(M) \neq 0 . c(M)$ is the "Dolbeault connectivity" of $M$.

THEOREM 7. Given $M$ as in Theorem 6 and given $r>0$, then $H^{p, q}(M) \rightarrow$ $\pi^{p, q}(M)$ is an isomorphism for $p+q \leqslant \delta(r)+c(M)$ and $q / p \geqslant r$.

Proof. Replace $\mathcal{E}_{M}^{* * *}$ by a minimal model (see Bousfield-Gugenheim [3, Definition 7.2]). By Proposition 7.3 of [3] the differential in $\mathfrak{N}$ is decomposable.

Consider the subalgebra $\Re$ generated by all the generators of $\mathfrak{N}$ with total degree less than $\delta(r)+c(M)$ and all those generators of $\Re$ with total degree equal to $\delta(r)+c(M)$ and bidegree $(p, q)$ with $q / p>r$. Notice that $\Re$ is a differential subalgebra of $\Re$. From our construction of $\Re$ it is clear that $\pi^{p, q}(\mathscr{T}) \rightarrow \pi^{p, q}(\Re)$ and $H^{p, q}(\mathscr{T}) \rightarrow H^{p, q}(\mathscr{T})$ are isomorphisms for the pairs $(p, q)$ we are considering. Hence it suffices to prove the result for $\Re$.

Consider the subalgebra $\mathcal{Q}$ of $\mathcal{\Re}$ generated by all generators of $\mathcal{N}$ having bidegree $(p, q)$ with $q / p \geqslant r$. Notice that $\mathscr{Q}$ is a differential subalgebra; in fact, $\mathbb{Q}$ is a DBA retract of $\Re$. Hence it suffices to prove our theorem for $\mathbb{Q}$. But clearly $\delta(r) \geqslant c(M)+1$ and Lemma 2 implies the theorem for $\mathbb{Q}$.

7. The cohomotopy of Kähler manifolds. An important class of examples of strictly formal manifolds are the compact Kähler manifolds. Deligne, Griffiths, Morgan, and Sullivan [6] have proved that any compact, connected Kähler manifold is de Rham formal. We improve this to

THEOREM 8. Any compact, connected Kähler manifold is strictly formal. In fact, we prove this for any manifold satisfying the ว̀-lemma.

We say that a manifold $M$ satisfies the $\partial \bar{\partial}$-lemma, if given any $x \in \mathcal{E}_{M}^{* * *}$ with $\partial x=\bar{\partial} x=0$ and with either $x=\partial y$ or $x=\overline{\partial y}$ for some $y$, then there exists a $z$ such that $x=\partial \bar{\partial} z$.

For the proof of Theorem 8, notice that any compact Kähler manifold satisfies the $\partial \bar{\partial}$-lemma. This is mentioned in [6] but the reader can use Lemmas 5.11 and 5.15 of [6] to prove it.

Now given any $M$ satisfying the $\partial \bar{\partial}$-lemma, define $\mathcal{K}_{M}^{* * *}$ to be the sub DBA 
of $\mathcal{E}_{M}^{* * *}$ given by the kernel of $\partial$. The inclusion $\left(\mathcal{K}_{M}^{* * *}, \bar{\partial}\right) \subset\left(\mathcal{E}_{M}^{* * *}, \bar{\partial}\right)$ is a weak equivalence. This is proved exactly like the proof by the $d^{c}$-diagram method on p. 270 of [6].

Choose a cofibrant model $\Re$ for $\left(\mathcal{K}_{M}^{* * *}, \bar{\partial}\right)$. A $\partial$ differential can be defined in $\Re$ as the zero map, and then the map $\mathscr{T} \rightarrow \mathcal{K}_{M}^{* *} \rightarrow \mathcal{E}_{M}^{* * *}$ becomes a map of BBA's. $M$ is clearly $\partial$-degenerate. There is an obvious map $\left(\mathcal{K K}_{M}^{* *}, \bar{\partial}\right) \rightarrow$ $\left(H_{\partial}^{*, *}(M), \bar{\partial}\right)$ where $H_{\partial}^{*, *}(M)$ denotes cohomology of $\mathcal{E}_{M}^{* * *}$ with respect to the $\partial$ differential. $\bar{\partial}$ on $H_{\partial}^{* * *}$ is the zero map and the map $\left(\mathcal{K}_{M}^{* *}, \bar{\partial}\right) \rightarrow$ $\left(H_{\partial}^{* * *}(M), 0\right)$ is a weak equivalence as the reader can check using p. 270 of [6] as a guide. Since $H^{*, *}(M) \cong H_{\partial}^{*, *}(M)$ as algebras (we have constructed an isomorphism via $\left.\left(H_{\partial}^{*, *}(M), 0\right) \leftarrow\left(\mathcal{K}_{M}^{* * *}, \bar{\partial}\right) \rightarrow\left(\mathcal{E}_{M}^{* * *}, \bar{\partial}\right)\right)$ it is clear that $M$ is strictly formal.

To state Theorem 9 we first define two functors from DBA's to DGA's. $D^{*}(\mathbb{Q})=\bigoplus_{r} \pi^{r, *-r}(\mathbb{Q})$ and $\mathcal{C}^{*}(\mathbb{Q})=\bigoplus_{r} H^{r, *-r}(\mathbb{Q}) . D^{*}(M)=D^{*}\left(\mathcal{E}_{M}^{* * *}\right)$ and $\mathcal{H}^{*}(M)=\mathcal{H}^{*}\left(\mathcal{E}_{M}^{* * *}\right), D^{*}(M)$ is a graded Whitehead coalgebra.

TheOREM 9. A d-degenerate manifold has a natural "Hodge" decomposition. To wit, in the category of $\partial$-degenerate manifolds, there exist a natural isomorphism of cohomotopy theories $D^{*}$ and $\pi^{*}$ and a natural isomorphism of cohomology theories $\mathcal{H}^{*}$ and $H^{*}$.

REMARKs. The above equivalences are compatible with the Hurewicz maps. The equivalence of $D^{*}$ and $\pi^{*}$ is as Whitehead coalgebras. Given an analytic fibre space which satisfies the hypothesis of Theorem 3, whose total space satisfies the $\partial \bar{\partial}$-lemma, and whose base is $\partial$-degenerate it is not hard to prove, using the techniques used in the proof of Theorem 3, that the fibre is $\partial$-degenerate. These techniques also show that the long exact cohomotopy sequence for $D^{*}$ is equivalent to the one for $\pi^{*}$.

Proof of Theorem 9. Since $\varepsilon_{M}^{* * *}$ is $\partial$-degenerate, there exists a cofibrant model $\mathfrak{N}$ with a zero $\partial$ differential, and a weak equivalence of BBA's $(\Re, \bar{\partial}) \rightarrow\left(\mathcal{E}_{M}^{* * *}, \bar{\partial}\right)$.

Since this is a map of BBA's, we get a map $(\mathscr{T}, d) \rightarrow\left(\mathcal{E}_{M}^{* * *}, d\right)$, which the cohomology Fröhlicher spectral sequence shows to be a weak equivalence. The complex $(\mathfrak{N}, d)$ is the complex $(\mathfrak{T}, \bar{\partial})$ and so we get isomorphisms of $\mathcal{H}^{*}(M)$ with $H^{*}(M)$ and $D^{*}(M)$ with $\pi^{*}(M)$. To show that these isomorphisms are independent of the choice of $\mathfrak{M}$ and are natural requires a bigraded version of results like 6.4 of Bousfield and Gugenheim [3]. Details are omitted.

We now prove a nice result on the Eilenberg-Moore spectral sequence.

THEOREM 10. Let 


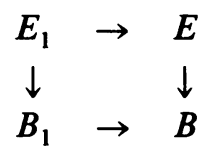

be a fibre square satisfying the hypotheses of Theorem 3. Suppose $E, B$, and $B_{1}$ are compact Kähler. Then $\mathcal{E}_{E_{1}}^{* * *}$ has the weak homotopy type of $B\left(H^{*, *}\left(B_{1}\right)\right.$, $\left.H^{*, *}(B), H^{*, *}(E)\right)$ and is hence a "formal consequence" of the cohomology rings of $B_{1}, B$, and $E$, and of the maps among them. In particular

$$
H^{r, s}\left(E_{1}\right) \cong \bigoplus_{p} \operatorname{Tor}_{H_{* * * *}^{p, r, s}-p}\left(H^{*, *}\left(B_{1}\right),\left(H^{*, *}(E)\right),\right.
$$

where $p$ runs over all the nonpositive integers. The isomorphism is a natural algebra isomorphism.

ReMARKs. There is a similar result for de Rham cohomology if $E, B$, and $B_{1}$ are compact Kähler.

With the hypotheses as in Theorem 10, there is the obvious result for the antiholomorphic homotopy type of $E_{1}$. The last remark after Theorem 9 generalizes as follows. Suppose $B_{1}$ and $E$ satisfy the $\partial \bar{\partial}$-lemma and that $B$ is $\partial$-degenerate. Then $E_{1}$ is $\partial$-degenerate.

ProOF OF THEOREM 10. In the proof of Theorem 8, we remarked that there were weak equivalences $\left(H_{\partial}^{*, *}(M), 0\right) \leftarrow\left(\mathcal{K}_{M}^{* *}, \bar{\partial}\right) \rightarrow\left(\mathcal{E}_{M}^{* * *}, \bar{\partial}\right)$. From this and Theorem 3 and its proof, Theorem 10 follows.

Formality has other consequences. If a subalgebra of the cohomology of a Dolbeault formal space is an algebra retract, then the functorial cofibrant model for the subalgebra is a retract of the one for the space. This gives theorems such as

THEOREM 11. For a Dolbeault formal manifold, the antiholomorphic cohomotopy is a formal consequence of the antiholomorphic cohomology. The holomorphic cohomology determines a formal fan of cohomotopy.

Proof. Clearly $H^{0, *}(M)$ is a retract of $H^{*, *}(M)$. The first part of the theorem follows from the observation just before the statement of Theorem 11.

Equally clearly, $H^{*, 0}(M)$ is a retract of $H^{*, *}(M)$, so the cohomotopy of the DBA $H^{*, 0}(M)$ is a retract of $\pi^{*, *}(M)$. Recall that formal fan was defined (before Theorem 6) to be the cohomotopy of $H^{*, 0}(M)$.

In fact, both the antiholomorphic cohomotopy and the holomorphic fan are retracts of $\pi^{*, *}$ as Whitehead coalgebras. Also notice that if $H^{*, 0}$ or $H^{0, *}$ has no products, the resulting cohomotopy is the injective Whitehead coalgebra on $H^{*, 0}$ or $H^{0, *}$. This is either $0, \mathrm{C}, \mathrm{C} \oplus \mathrm{C}$, or a countably infinite dimensional vector space. This may be seen by observing that the calculation is the same as for a wedge of spheres.

The chief import of Theorem 11 is that we can work out Whitehead 
coalgebra summands of the Whitehead coalgebra solely from knowledge of the holomorphic or antiholomorphic cohomology. These Whitehead coalgebras are summands of the de Rham Whitehead coalgebra, and so the theorem can be proved from the results in [6] tensored with $\mathbf{C}$.

Hurewicz theorems for formal spaces are very strong as a consequence of the algebraic

THEOREM 12. Let $\mathbb{Q}$ be a DBA with $\bar{\partial}=0$. Then $Q^{*, *} \mathbb{Q} \rightarrow \pi^{* * *} \mathbb{Q}$ is a monomorphism. A similar result holds for DGA's.

Proof. Choose a map $S(Q \mathbb{Q}) \rightarrow \mathbb{Q}$ and a cofibrant model $\Re \rightarrow \mathbb{Q}$ which is surjective. $S(Q Q)$ is cofibrant, so we get a map $S(Q \mathscr{Q}) \rightarrow \mathfrak{\pi}$. This splits the natural map $Q \Re \rightarrow Q Q$, so the theorem follows.

One consequence of Theorem 12 and Sullivan's theory [15] is that the map from $\pi_{*}(X) \otimes Q$ to the primitives in $H_{*}(X ; Q)$ is a surjection for any formal space $X$ with $\pi_{1}(X)$ nilpotent.

For Kähler manifolds, one obtains the following corollary.

COROllary 3. If $M$ is compact, connected Kähler, then the kernel of the Hurewicz map in bidegree $(p, q)$ is isomorphic to the kernel of the Hurewicz map in bidegree $(q, p)$.

Proof. $H^{p, q} \cong H^{q, p}$ for a compact Kähler manifold [21, p. 189]. Hence $Q H^{p, q} \cong Q H^{p, q}$ since the isomorphism is a ring map.

The corollary implies that rank of the integral Hurewicz homomorphism is even in the odd dimensions of a compact Kähler manifold. Of course, the corollary can be proved directly from the theory in [6] with more work.

As an example of the use of the corollary, consider $M=U(n) / T$, the unitary group modulo a maximal torus. We shall show that $H^{*}(M ; Q)$ is generated by the 2 dimensional classes. The rational homotopy of $M$ is easily worked out. There is an $n$ dimensional vector space in dimension 2 and a copy of $Q$ in each odd dimension above one up to dimension $2 n-1$. By the corollary $\pi_{2 r-1}(M) \otimes Q \rightarrow H_{2 r-1}(M ; Q)$ is the zero map since $M$ is Kähler [2, Proposition 14.6]. $Q H^{*} \rightarrow \pi^{*}$ is monic by Theorem 10, so $Q H^{*}$ is all in dimension 2 as claimed. In fact, $Q H^{*, *}(M)=Q H^{1,1}(M)$ since, if not, Theorem 11 implies that there is some odd dimensional cohomotopy on the antiholomorphic line and in the formal fan. This can be seen to force $\operatorname{dim} \pi^{2 r+1}(M) \geqslant 2$ for some $r$ in this case.

We conclude this section with some calculations for a complete intersection $V_{n}$ in $P^{n+r} . V_{n}$ is algebraic, hence Kähler, hence strictly formal. The Dolbeault cohomology of $V_{n}$ has been completely determined by Hirzebruch [11] as a consequence of the Riemann-Roch theorem. Except for powers of the Kähler form (which, of course, is of type $(1,1)$ ) a complete intersection has no cohomology up to real dimension $n$. These give the simplest examples 
known to us of manifolds whose $\pi^{p, q}$ has negative $q$. To wit, a nonsingular hypersurface in $P^{n+1}$ of degree $a$ has $h^{0, n}=h^{n, 0}=\left(\begin{array}{c}a-1 \\ n+1\end{array}\right)$, which is bigger than 1 if $a$ is at least $n+3$. Hence the holomorphic fan in this case has elements in $\pi^{p, q}$ with $q$ arbitrarily small.

Since these manifolds are compact, we can dualize $\pi^{*}$ to get homotopy information. In particular, a complete intersection has two disjoint, free, integral Whitehead algebras in its ordinary homotopy, each of which has $h^{0, n}$ generators. These are often infinitely generated as an abelian group.

We can actually do better. Consider the subalgebra of $H^{*, *}(M)$ generated by all the forms of bidegree $(p, q)$ for $p>q$. This is seen to be a subalgebra retract for complete intersections. All products are 0 , so this generates a Whitehead coalgebra, which dualizes down to give a free Whitehead algebra in ordinary homotopy. There is a disjoint, free Whitehead algebra of the same size coming from the subalgebra generated by the forms of bidegree $(p, q)$ for $p<q$.

We close this paper with a deeper analysis of the complex homotopy groups of a compact $n$ dimensional Kähler manifold $M$. Write $\mathcal{H}=$ $\bigoplus H^{r}(M)=\bigoplus H^{p, q}(M)$ and write $\pi_{*}(M) \otimes C$ for the dual vector space to $\pi^{*}(M)$. If $M$ is nilpotent, this agrees with the classical meaning for $\pi_{*}(M) \otimes$ C. If $\mathscr{Q}$ is any DGA, we shall write $\pi_{*}(\mathscr{Q})$ for the dual vector space to $\pi^{*}(\mathbb{Q})$. Since $M$ is de Rham formal, $\pi_{*}(M) \otimes C$ is isomorphic to $\pi_{*}(\mathcal{H})$ as Whitehead algebras.

For all $m \leqslant n$, we define the $m$ dimensional Hodge level of $M$ to be $l_{m}=\sup \left\{|q-p|: h^{p, q} \neq 0, p+q=m\right\}$. We define

$$
r_{m}=\log \left[\left(m+l_{m}\right) /\left(m-l_{m}\right)\right]
$$

with the understanding that $r_{m}=-\infty$ if $l_{m}=-\infty$.

Let $u=\max \left\{r_{m}: m<n\right\}$ and let $\mathcal{S}$ be the subalgebra of $\mathcal{H}$ generated by $H^{p, q}$ where $p+q=\dot{n}$ and $|\log q / p|>u$. It follows from Poincaré duality and the fact that $H^{p, q}=\bar{H}^{q, p}$ that $\overline{\mathcal{S}}$ is an ideal in $\mathcal{H}$.

For any ideal $\mathscr{G}$ in a connected finite type graded algebra $\mathcal{Q}$, we define the ideal spectral sequence as follows. Filter $\mathbb{Q}$ by the powers of $\mathcal{G}$, that is, $F^{0}=\mathscr{Q}, F^{1}=\mathscr{G}, F^{2}=\mathscr{Q}^{2}, \ldots$ Proposition 1 in $\$ 3$ gives a spectral sequence of Whitehead coalgebras with $E_{1}$ term given by $\pi^{*, *}\left(E_{0} \mathscr{Q}\right)$ and abutment $\pi^{*}(\mathbb{Q})$. If $\mathbb{Q}^{1}=0$, this spectral sequence converges. The ideal spectral sequence is the vector space dual to this spectral sequence. It is a spectral sequence of Whitehead algebras with $E^{1}$ term given by $\pi_{* *}\left(E_{0} \mathbb{Q}\right)$ and abutment $\pi_{*}(\mathbb{Q})$. There is an edge homomorphism $\pi_{*}(\mathscr{Q} / \mathscr{G}) \rightarrow E_{0, *}^{1}=$ $\pi_{0, *}\left(E_{0} \mathscr{Q}\right)$.

THEOREM 13. With the above notation, $E^{1}=E^{\infty}$ in the ideal spectral sequence for $\overline{\mathcal{S}}$ in $\mathcal{H}$. 
Proof. The theorem is trivial if $\bar{\delta}=0$. So assume $\bar{\delta} \neq 0$.

Let $\mathscr{D}$ be the subalgebra of $\mathcal{H}$ generated by $H^{p, q}$ where $p+q \leqslant n$ and $|\log q / p| \geqslant u$. It is clear from the Lefschetz decomposition [21] of $\mathcal{H}$ that

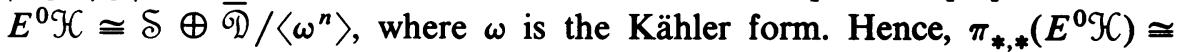
$\pi_{*}(\delta) \bigvee \pi_{*}\left(\mathscr{D} /\left\langle\omega^{n}\right\rangle\right)$, where $\vee$ denotes the free product of Whitehead algebras. This is a consequence of the fact that $\delta \oplus \mathscr{D} /\left\langle\omega^{n}\right\rangle$ is the algebra analogue of the one point union of topological spaces.

Let $X$ be a connected sum of $k$ copies of $S^{n} \times S^{n}$, where $k=\Sigma_{q / p>u} h^{p, q}$. By Proposition 4.4 of [16], $X$ is de Rham formal. Since $H^{*}(X ; \mathrm{C})$ is isomorphic to $\delta, \pi_{*}(\delta)$ is isomorphic to $\pi_{*}(X) \otimes \mathbf{C}$.

LEMMA 3. There is a filtration on $\pi_{*}(\delta)$ such that $E^{0} \pi_{*}(\delta)$ is isomorphic to $\pi_{*}(Y) \otimes C$ where $Y$ is the one point union of $S^{n} \times S^{n}$ and a bouquet of $2 k-2$ copies of $S^{n}$.

To prove Lemma 3, let $\delta_{1}=H^{*}\left(S^{n} \times S^{n} ; \mathrm{C}\right)$ and embed $\delta_{1}$ as an ideal in $\delta$ so that $\delta / \overline{\mathcal{S}}_{1}$ is isomorphic to the cohomology ring of a bouquet of spheres. In the ideal spectral sequence for $\delta_{1}$ in $\delta, E_{1}=E^{\infty}$ since $E^{1}=$ $\pi_{*}\left(\delta / \delta_{1}\right) \bigvee \pi_{*}\left(\delta_{1}\right) ; \pi_{*}\left(\delta / \delta_{1}\right)$ consists of infinite cycles by the edge homomorphism and $\pi_{*}\left(\mathcal{S}_{1}\right)=\pi_{*}\left(S^{n} \times S^{n}\right) \otimes C$ is generated by infinite cycles by Theorem 12. This proves the lemma.

In the ideal spectral sequence for $\bar{\delta}$ in $\mathcal{H}, \pi_{*}\left(\mathscr{D} /\left\langle\omega^{n}\right\rangle\right)$ consists of infinite cycles by the edge homomorphism and $\pi_{*}(\delta)$ is generated by infinite cycles by Lemma 3 and Theorem 12. Hence, $E^{1}=E^{\infty}$.

As a corollary of the proof of Theorem 13, we get the following.

COROLlaRY 4. If $M$ is a simply connected compact Kähler manifold with $r_{n}>u$, then $\pi_{*}(M) \otimes C$ is isomorphic as a graded vector space to both of the following:

(1) the free product of $\pi_{*}\left(\mathscr{D} /\left\langle\omega^{n}\right\rangle\right)$ and $\pi_{*}(X) \otimes \mathbf{C}$ where $X$ is a connected sum of $\sum_{\log q / p>u} h^{p, q}$ copies of $S^{n} \times S^{n}$.

(2) the free product of $\pi_{*}\left(\mathscr{D} /\left\langle\omega^{n}\right\rangle\right), \pi_{*}\left(S^{n} \times S^{n}\right) \otimes \mathbf{C}$, and the complex homotopy groups of a bouquet of $\sum_{|\log q / p|>u} h^{p, q}-2$ copies of $S^{n}$.

Because $\mathscr{D}=$ the diagonal cohomology $\bigoplus H^{p, p}(M)$ whenever $M$ is a complete intersection, we get the following almost complete computation of the complex homotopy of complete instructions.

THEOREM 14. If $M$ is a complete intersection of complex dimension $n>2$, then $M$ is simply connected and, if $l_{n} \geqslant 1, M$ has the same complex homotopy groups as the one point union of $S^{n} \times S^{n}, \mathbf{C} P^{n-1}$, and $m$ copies of $S^{n}$ where $m$ is $\left(\sum_{p+q=n} h^{p, q}\right)-2$ if $n$ is odd, $\left(\sum_{p+q=n} h^{p, q}\right)-3$ if $m$ is even.

In Theorem 14, we have reproduced the main computation in [15] without using the complicated machinery of differential graded Lie algebras. 
The case of Hodge level $l_{n}<1$ is not covered by Theorem 14. But Rapaport [18] has given a table of complete intersections with $l_{n}<2$. Those with $l_{n}<1$ are $V_{n}(2), V_{n}(2,2)$, and $V_{2}(3)$ where $V_{n}\left(a_{1}, \ldots, a_{r}\right)$ denotes an $n$ dimensional complete intersection defined by $r$ equations of degrees $a_{1}, \ldots, a_{r}$. These remaining cases can be handled one by one. See [15] for details.

For a nonsingular hypersurface $V_{n}(d)$, the integer $m$ in Theorem 14 is easily computed. If $n$ is even, $\Sigma_{p+q=n} h^{p, q}$ is $d^{-1}\left\{(d-1)^{n+2}-1\right\}+2$; if $n$ is odd, it is $d^{-1}\left\{(d-1)^{n+2}+1\right\}-1$. See [23].

\section{BIBLIOGRAPHY}

1. A. Borel, A spectral sequence for complex analytic bundles, Topological Methods in Algebraic Geometry, Springer-Verlag, New York, 1966.

2. A. Borel and F. Hirzebruch, Characteristic classes and homogeneous spaces. I, Amer. J. Math. 80 (1958), 458-538.

3. A. K. Bousfield and V. K. A. M. Gugenheim, On PL de Rham theory and rational homotopy type, Mem. Amer. Math. Soc., No. 179, 1976.

4. E. Calabi and B. Eckmann, A class of compact complex manifolds, Ann. of Math. (2) 58 (1953), 494-500.

5. H. Cartan, Algèbres d'Eilenberg-Mac Lane et homotopie, Exposé 3, Séminaire H. Cartan de l'École Norm. Sup., Secrétariat Mathématique, Paris, 1954/1955.

6. P. Deligne, P. Griffiths, J. Morgan and D. Sullivan, Real homotopy theory of Kähler manifolds, Invent. Math. 29 (1975), 245-274.

7. S. Eilenberg and J. C. Moore, Limits and spectral sequences, Topology 1 (1962), 1-23.

8. A. Fröhlicher, Relations between the cohomology groups of Dolbeault and topological invariants, Proc. Nat. Acad. Sci. U.S.A. 41 (1955), 641-644.

9. H. Grauert, On Levi's problem and the imbedding of real analytic manifolds, Ann. of Math. (2) 68 (1958), 460-472.

10. R. C. Gunning and H. Rossi, Analytic functions of several complex variables, Prentice-Hall, Englewood Cliffs, N.J., 1965.

11. F. Hirzebruch, Der Satz von Riemann-Roch und das Toddsche arithmetische Geschlecht für algebraische Mannigfaltigkeiten (Proc. Internat. Congr. Math., Amsterdam, 1954), Noordhoff, Groningen, North-Holland, Amsterdam, 1956.

12. D. Husemoller, J. C. Moore and J. Stasheff, Differential homological algebra and homogeneous spaces, J. Algebra 5 (1974), 113-185.

13. S. Mac Lane, Homology, Springer-Verlag, New York, 1963.

14. J. P. May, A general algebraic approach to Steenrod operations, Steenrod Algebra and its Applications, edited by Frank Peterson, Lecture Notes in Math., vol. 168, Springer-Verlag, New York, 1970.

15. J. Neisendorfer, The rational homotopy groups of complete intersections (preprint).

16. J. Neisendorfer and T. Miller, Formal and coformal spaces (preprint).

17. D. Quillen, Rational homotopy theory, Ann. of Math. (2) 90 (1969), 205-295.

18. M. Rapaport, Complément à l'article de P. Deligne "La conjecture de Weil pour les surfaces K3," Invent. Math. 15 (1972), 227-236.

19. L. Smith, Homological algebra and the Eilenberg-Moore spectral sequences, Trans. Amer. Math. Soc. 129 (1967), 58-93.

20. D. Sullivan, Topology of manifolds and differential forms, (Proc. Conf. Manifolds, Tokyo, 1973).

21. R. O. Wells, Differential analysis on complex manifolds, Prentice Hall, Englewood Cliffs, N.J., 1973. 
22. G. W. Whitehead, On mappings into group-like spaces, Comment. Math. Helv. 28 (1954), 320-328.

23. R. S. Kulkarni and J. W. Wood, Topology of non-singular complex hypersurfaces (preprint). DePartment of Mathematics, Syracuse University, Syracuse, New York 13210

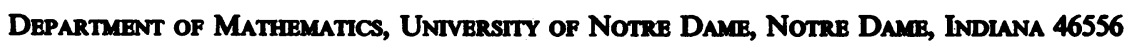

Current address: Department of Mathematics, Fordham University, Bronx, New York 10458 